\title{
ARTICLE
}

Received 27 Jul 2014 | Accepted 3 Mar 2015 | Published 16 Apr $2015 \quad$ DOl: 10.1038/ncomms7841

\section{Dissociation of Bak $\alpha 1$ helix from the core and latch domains is required for apoptosis}

Amber E. Alsop ${ }^{1,2}$, Stephanie C. Fennell ${ }^{1}$, Ray C. Bartolo ${ }^{1}$, Iris K.L. Tan ${ }^{1}$, Grant Dewson ${ }^{1,2}$ \& Ruth M. Kluck ${ }^{1,2}$

During apoptosis, Bak permeabilizes mitochondria after undergoing major conformational changes, including poorly defined $\mathrm{N}$-terminal changes. Here, we characterize those changes using 11 antibodies that were epitope mapped using peptide arrays and mutagenesis. After Bak activation by Bid, epitopes throughout the $\alpha 1$ helix are exposed indicating complete dissociation of $\alpha 1$ from $\alpha 2$ in the core and from $\alpha 6-\alpha 8$ in the latch. Moreover, disulfide tethering of $\alpha 1$ to $\alpha 2$ or $\alpha 6$ blocks cytochrome c release, suggesting that $\alpha 1$ dissociation is required for further conformational changes during apoptosis. Assaying epitope exposure when $\alpha 1$ is tethered shows that Bid triggers $\alpha 2$ movement, followed by $\alpha 1$ dissociation. However, $\alpha 2$ reaches its final position only after $\alpha 1$ dissociates from the latch. Thus, $\alpha 1$ dissociation is a key step in unfolding Bak into three major components, the $N$ terminus, the core $(\alpha 2-\alpha 5)$ and the latch $(\alpha 6-\alpha 8)$.

\footnotetext{
${ }^{1}$ The Walter and Eliza Hall Institute of Medical Research, Parkville, Victoria 3052, Australia. ${ }^{2}$ Department of Medical Biology, University of Melbourne, Parkville, Victoria 3052, Australia. Correspondence and requests for materials should be addressed to R.M.K. (email: kluck@wehi.edu.au).
} 
B ak and Bax are pro-apoptotic members of the Bcl-2 protein family that controls apoptosis ${ }^{1,2}$. In healthy cells, Bak and Bax exist in inert conformations in which their hydrophobic $\alpha 5$ helices are surrounded by seven or eight amphipathic helices ${ }^{3,4}$. A carboxy (C)-terminal helix, $\alpha 9$, anchors Bak constitutively in the mitochondrial outer membrane $(\mathrm{MOM})^{5,6}$, whereas in Bax, the $\alpha 9$ helix is initially sequestered in the hydrophobic surface groove, rendering Bax largely cytosolic ${ }^{4,7}$. When cells experience sufficient stress, the $\mathrm{BH} 3$-only class of Bcl-2 family proteins cause Bak and Bax to undergo a series of conformation changes-collectively referred to as 'activation'-that culminate in dimerization, leading to pore formation in the $\mathrm{MOM}^{8-10}$. Pore formation releases cytochrome $c$ into the cytosol to initiate caspase activation and dismantle the cell ${ }^{11}$.

Bak activation is triggered by binding of $\mathrm{BH} 3$-only proteins (for example, Bid or Bim) to a hydrophobic groove on the Bak surface $^{11,12}$. Two major consequences are exposure of the $\mathrm{BH} 3$ domain in $\alpha 2$ (ref. 9) and exposure of epitopes in the Bak amino (N) terminus ${ }^{10,13}$. The $\mathrm{N}$ terminus comprises a semi-structured $\mathrm{N}$-segment (aa1-23) ${ }^{14}$ and the $\alpha 1$ helix (aa24-50) containing the $\mathrm{BH} 4$ domain $^{15}$. Similar conformation changes occur in Bax during its activation ${ }^{16-18}$. Another key conformation change identified in Bak and Bax is separation of the latch $(\alpha 6-\alpha 8)$ from the core $(\alpha 2-\alpha 5)^{19,20}$. After these large changes in conformation, the proteins form $\mathrm{BH} 3$ :groove dimers and make significant contact with the outer membrane surface ${ }^{9,16,19,21,22}$.

Elucidating the structures of the fully activated forms of Bak and Bax will provide insight into how these proteins self-associate to form the apoptotic pore. However, the Bak N-terminal epitopes exposed by activation are poorly mapped so it remains unclear whether some or all elements of the N-termini re-position during activation, or whether they are exposed by re-positioning of other elements, for example, the $\alpha 6-\alpha 8$ latch domain ${ }^{19}$ or by dissociation of Bak from other proteins such as VDAC2 (refs 10,23). To gain insight into the molecular changes occurring at the Bak $\mathrm{N}$ terminus during apoptosis, we mapped the epitopes of 11 anti-Bak antibodies using peptide arrays and mutagenesis. We also developed a novel fluorescence-activated cell sorting (FACS) protocol for measuring epitope exposure after disulfide tethering, which allowed us to examine the sequence of Bak conformation changes during activation.

Herein, we show that N-terminal epitopes of Bak are mostly linear, and that many are located within the $\alpha 1$ helix including two that overlap with the $\mathrm{BH} 4$ domain of Bak. All epitopes within $\alpha 1$ and the $\mathrm{N}$-segment are conformation specific as their exposure increases upon Bak activation. Intramolecular tethering demonstrated that separation of $\alpha 1$ and $\alpha 6$, and of both from the core, is required for pore formation. In addition, dissociation of $\alpha 1$ from $\alpha 2$ is required for $\mathrm{BH} 3$ :groove dimer formation. Thus dissociation of $\alpha 1$ from both the core and latch domains is a key event in the activation of Bak.

\section{Results}

Most Bak antibodies have linear epitopes. To investigate Bak $\mathrm{N}$-terminal conformation change during apoptosis, we compared the binding properties of 11 anti-Bak antibodies. Table 1 lists known characteristics of the antibodies, together with the key residues involved in their epitopes, as determined below.

Antibodies were first tested by SDS-polyacrylamide gel electrophoresis (SDS-PAGE) and western blotting to determine if their epitopes were linear (also called continuous or sequential epitopes) or assembled (also called discontinuous or conformational epitopes $)^{24}$. As all antibodies recognized human Bak after SDS-PAGE (Fig. 1a), each epitope was linear.
To gain insight into the possible location of the epitopes, we also tested each antibody for its ability to bind mouse Bak, since the mouse Bak protein sequence is $77 \%$ similar to human Bak and the antibodies were unlikely to bind in regions of sequence divergence. Six antibodies (14-36, NT, aa23-38, G317-2, 4B5 and G23) also recognized mouse Bak (Fig. 1a). For three of these antibodies (14-36, NT and aa23-38), the immunogen contained a stretch of residues (26-36) conserved in mouse and human Bak (Table 1). Another two antibodies (4B5 and G23) had previously been mapped to a conserved region ${ }^{9}$.

Epitopes map to various sites in the Bak $\mathbf{N}$ terminus. As the Bak epitopes were linear, further mapping was performed using peptide arrays. When applied to an array of overlapping 15-mer peptides from human Bak, all antibodies bound to at least one peptide (Fig. 1b). When applied to a similar array of mouse Bak peptides, the six antibodies that bound mouse Bak on western blots (Fig. 1a) also recognized at least one peptide (Supplementary Fig. 1).

Epitope mapping by peptide array was consistent with the immunogen sequence and previous mapping data (Table 1), and together the panel of antibodies recognized at least five distinct epitopes in the $\mathrm{N}$ terminus (Fig. 1b). Note, the $\mathrm{N}$-segment positions of the 2-14 and 8F8 epitopes suggested by the array were confirmed by western blotting of $\mathrm{N}$-terminally truncated Bak (Fig. 1c).

As seven antibodies (14-36, NT, a23-38, G317-2, Ab-1, Ab-2, 7D10) bound peptides containing $\alpha 1$ residues, we focused on mapping their epitopes further and designed an array of 8-mers with only a single residue offset to define the minimal set of residues required for their binding. As expected, due to their polyclonal nature the three peptide-derived antibodies (14-36, NT and aa23-38) produced complex binding patterns for this array (Supplementary Fig. 2), while the monoclonal antibodies (G317-2, Ab-1, Ab-2 and 7D10) produced simple binding patterns. G317-2 bound four peptides (Fig. 2a) refining its epitope to ${ }^{32} \mathrm{EEVFR}^{36}$, and suggesting that E32 and R36 are particularly important for its binding. Ab-1 and Ab-2 bound predominantly to the same peptide ${ }^{38}$ YVFYRHQQ ${ }^{45}$, suggesting their epitopes are very similar. The 7D10 epitope begins after $\alpha 1$, with ${ }^{51}$ GVAAP $^{55}$ the minimal set of residues required for binding. Thus, peptide arrays of increasing resolution defined the minimal linear epitopes of several antibodies to the Bak $N$ terminus.

The BH4 domain in $\alpha 1$ is critical for Bak stability. To analyse the role of $\alpha 1$ in Bak function, we utilized 10 variants of Bak in which residues (E25, A28, V34, F35, R36, S37, Y38, Y41, R42, Q44) were substituted with cysteine (Fig. 2b,c). After stable expression in $\mathrm{Bak}^{-/-} \mathrm{Bax}-/-\mathrm{MEF}$, most variants were present at levels similar to that of wild-type human Bak (Fig. 2c), indicating that cysteine substitution had not significantly altered protein expression or stability. The exceptions were three variants (V34C, F35C, Y38C) that were present at only low levels (Fig. 2c). Notably, these residues lie in the BH4 domain of Bak (Fig. 2b), the sequence motif present in all multidomain members of the Bcl-2 family, including viral prosurvival proteins ${ }^{15}$. The destabilizing effect of cysteine substitution within the $\mathrm{BH} 4$ domain is consistent with the high conservation of this motif ${ }^{15}$ and with observations that truncating Bak $\alpha 1$ results in low protein levels after retroviral expression ${ }^{14}$.

The $\mathrm{BH} 4$ domain contributes to the G317-2 and Ab-1 epitopes. The cysteine-substituted Bak variants were also used to test whether the residues within $\alpha 1$ epitopes were required for 


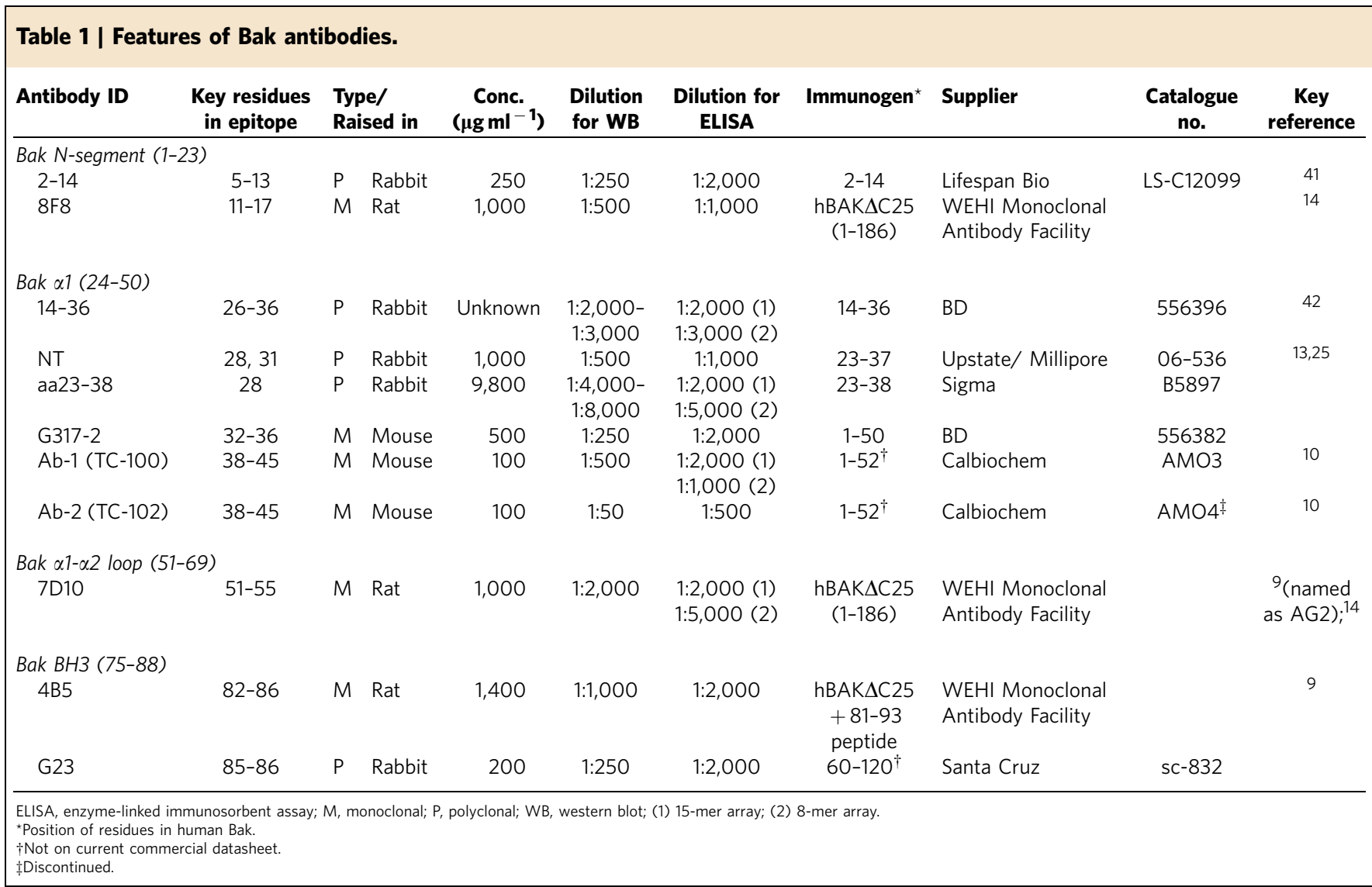

antibody binding (Fig. 2d). Among the polyclonal antibodies, both a23-38 and NT bound weakly to A28C (Fig. 2d), indicating that dominant epitopes among their collections of immunoglobulins overlap and that most depend on A28. The epitopes of 14-36 were also sensitive to mutation of A28 (Fig. 2d). Notably, each of the epitopes for the monoclonal antibodies relied on at least some residues within the $\mathrm{BH} 4$ domain $\left({ }^{34} \mathrm{VFRSYV}^{39}\right)$. G317-2 failed to bind V34C, F35C and R36C, consistent with its epitope being at EEVFR. Ab-1 and Ab-2 failed to bind $\mathrm{Y} 38 \mathrm{C}$, Y41C and R42C, consistent with their epitope being within YVFYRHQQ, and with a recent report that Y38 is critical for Ab-1 binding 25 . Thus, the anti-Bak antibodies tested bound to three distinct sites (residues 28, 34-36 and 37-44) along $\alpha 1$.

'N-terminal exposure' involves complete dissociation of $\alpha 1$. Antibodies generated to the Bak $\mathrm{N}$ terminus have often been used to monitor Bak conformation change (activation) during apoptosis ${ }^{10,13,14}$. In particular, $\mathrm{Ab}-1$ has been widely used as a 'conformation-specific' antibody to report 'N-terminal exposure' of Bak during apoptosis ${ }^{10}$. Based on our mapping of the Ab-1 epitope, two residues (Y38 and R42) required for Ab-1 binding are buried in the structure of non-activated Bak (Supplementary Fig. 3), arguing that this central region of $\alpha 1$ must become exposed during apoptosis. A similar argument holds for the start of $\alpha 1$, as the NT antibody recognizes only activated $\mathrm{Bak}^{13}$ and its epitope includes both A28 (Fig. 2d) and T31 25 , which are also buried in non-activated Bak (Supplementary Fig. 3). The $\mathrm{N}$-segment also undergoes conformation change, as 8F8 binds preferentially to activated $\mathrm{Bak}^{14}$. Thus, based on literature reports of three 'conformation-specific' antibodies (8F8, NT, Ab-1) and epitope mapping of those antibodies, at least three distinct sites
(11-17, 28-31 and 38-45) in the $\mathrm{N}$ terminus become exposed during Bak activation.

To further examine Bak N-terminal exposure during apoptosis, five additional $\mathrm{N}$-terminal antibodies were tested for immunoprecipitation of Bak before and after activation by Bid (Fig. 3). Three cell lines were used to compare recognition of endogenous mouse and human Bak with exogenous human Bak. All five antibodies bound to Bak more efficiently after its activation (Fig. 3). The 14-36 and G317-2 antibodies were most efficient in the conditions used (Fig. 3, compare bound and unbound fractions). The specificity of these additional five antibodies for an active conformation thus confirms that the entire $\mathrm{N}$ terminus (that is, the $\mathrm{N}$-segment and $\alpha 1$ ) dissociates from the remainder of the protein during Bak activation. Furthermore, when monitored by Ab-1 or G317-2 epitope exposure, this conformation change may be considered 'BH4 exposure', much as 4B5 or G23 epitope exposure signifies 'BH3 exposure'.

Dissociation of $\alpha 1$ from $\alpha 6$ is required for pore formation. To better understand how Bak unfolds during activation, we examined whether $\mathrm{BH} 4$ exposure occurs before or after $\mathrm{BH} 3$ exposure. This was done by disulfide tethering the $\mathrm{N}$ terminus to the remainder of Bak at three positions (Fig. 4a). One tether (WT) utilized the native cysteines in human Bak, C14 in the N-segment and C166 in the $\alpha 6-\alpha 7$ loop. Two other tethers (Y41C:A79C and A28C:L163C) involved cysteine substitutions at the relevant positions in cysteine-less human Bak (C14S/C166S). Each tether was induced with high efficiency following addition of the oxidizing agent copper phenanthroline (CuPhe), as indicated by faster migration of Bak during non-reducing SDS-PAGE (Fig. 4b). The mutations did not alter Bak function as indicated 


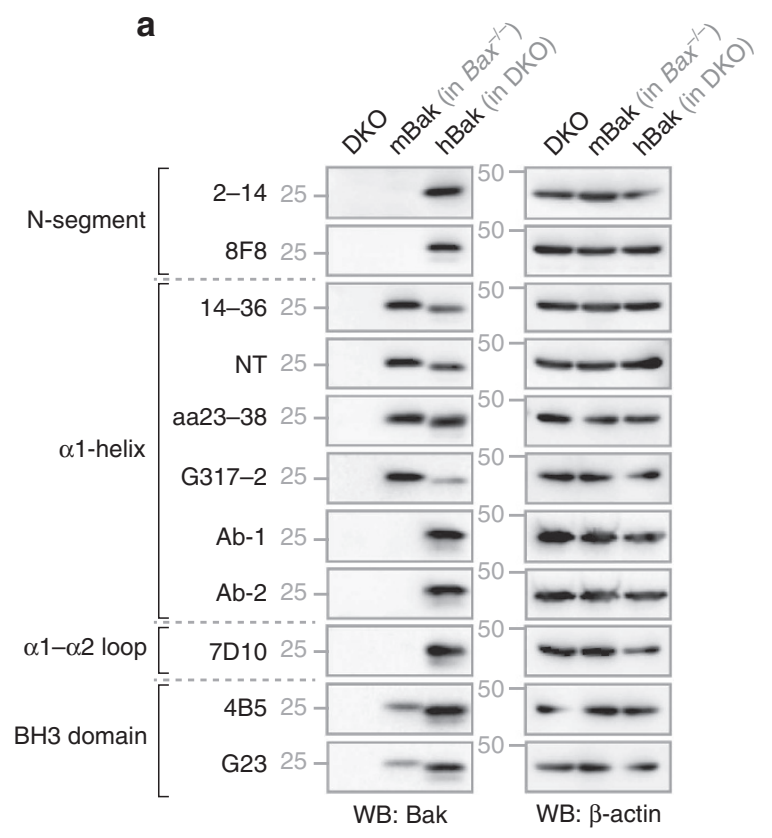

b
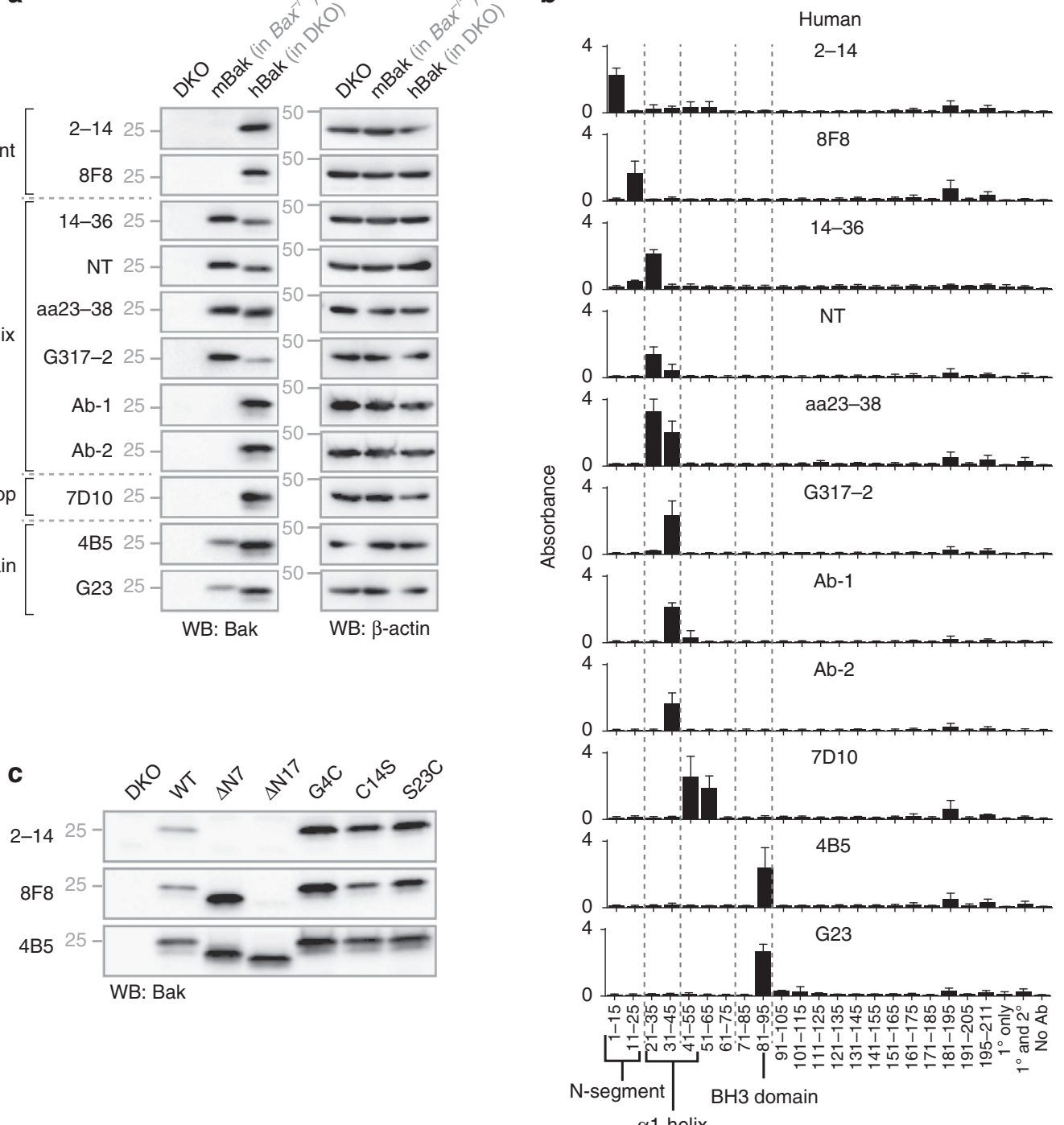

Figure 1 | Mapping N-terminal epitopes of Bak. (a) Bak antibodies recognize linear epitopes in human Bak, but only a subset recognize mouse Bak. Lysates from MEF expressing no Bak (Bax ${ }^{-/-} \mathrm{Bak}^{-/-}(\mathrm{DKO})$ ), mouse Bak (Bax ${ }^{-/-}$) or WT human Bak (in DKO) were analysed by western blot using the indicated antibodies. Blots were re-probed with $\beta$-actin to compare loading. Data are representative of two independent experiments. (b) Most Bak epitopes map to peptides from the $\mathrm{N}$ terminus. Histograms showing immunoreactivity of Bak antibodies towards biotinylated 15-mer hBak peptides, as determined by enzyme-linked immunosorbent assay. $X$ axis labels indicate residue number or control reaction conditions. Data are mean and s.d. of three independent experiments. (c) The epitopes of antibodies binding in the Bak N-segment are distinct. The 2-14 and 8F8, which bound to peptides corresponding to $\mathrm{N}$-segment residues in $\mathbf{b}$, were tested by western blot for their ability to bind $\mathrm{N}$-terminally truncated or single residue mutants of hBak (as indicated) expressed in DKO MEF. On the basis of loss of signal, residues 1-7 are required for the 2-14 antibody to bind Bak, whereas residues 8-17 are required for the $8 \mathrm{~F} 8$ antibody to bind Bak (as also shown in ref. 14). Binding by $4 \mathrm{~B} 5$ is shown as a reference for expression levels of various mutants, since its epitope in the $\mathrm{BH} 3$ domain is $\mathrm{C}$-terminal to the $\mathrm{N}$-segment ${ }^{9}$. Data are representative of two independent experiments.

by cytochrome $c$ in response to Bid (Fig. 4c). However, in contrast to $\mathrm{C} 14 \mathrm{~S} / \mathrm{C} 166 \mathrm{~S}$ Bak, when WT or cysteine double mutant Bak was incubated with $\mathrm{CuPhe}$ to induce tethers, cytochrome $c$ release no longer occurred in response to $\mathrm{Bid}, \mathrm{Bim} \mathrm{BH} 3$ peptide or heat (Fig. 4c). These data support a recent report that tethering WT Bak blocks pore formation in response to heat ${ }^{25}$, and defines $\alpha 1$ dissociation as required for Bak pore formation.

To assess which Bak conformation changes were hindered by these tethers, epitope exposure was quantified by flow cytometry. While Ab-1 is commonly used for flow cytometry of Bak, the Y41C substitution disrupted Ab-1 binding (Fig. 4d,e). Instead, we used G317-2, which only recognizes activated Bak (Figs 3 and $4 \mathrm{~d}$ ), and whose epitope also overlaps with the $\mathrm{BH} 4$ domain (Fig. 2d). After activation by Bid, the expected binding of G317-2 to all human Bak variants (Fig. 4e), as well as mouse Bak (Fig. 4f), was readily detectable by FACS. Thus, G317-2 is a particularly useful alternative to the Ab-1 antibody as it efficiently recognized activated forms of both human and mouse Bak by flow cytometry (and immunoprecipitation).

When $\alpha 1$ and $\alpha 2$ were tethered (Y41C:A79C) before cells being incubated with Bid, G317-2 bound to the same proportion of cells and with the same efficiency as the untethered samples (Fig. 5a,b). Thus, the tethered $\alpha 1$ and $\alpha 2$ helices were able to dissociate as a unit from the rest of the protein to expose the $\mathrm{BH} 4$ domain. In contrast, when $\alpha 1$ and $\alpha 6$ were tethered (A28C:L163C), G317-2 did not bind (Fig. 5a,b), indicating that the $\alpha 1-\alpha 6$ tether had prevented movement of $\alpha 1$. This is supported by Ab- 1 also failing to bind its epitope (Fig. 5d), which is located further away from 
a
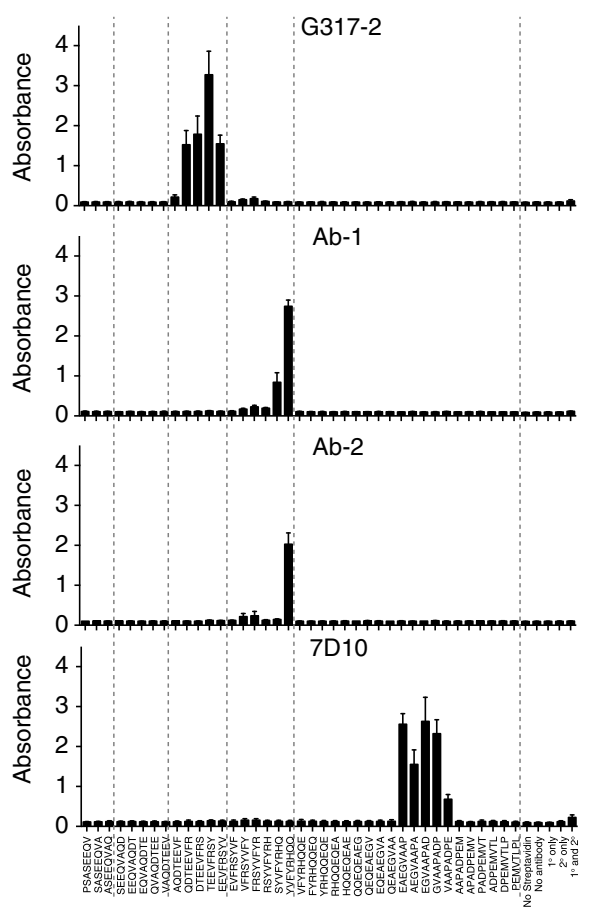

b

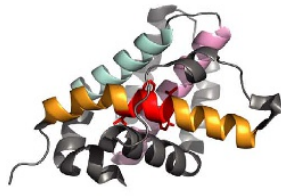
${ }^{24}$ EEQVAQDTEEVIFRYVFYRHQQEQEAE ${ }^{50}$

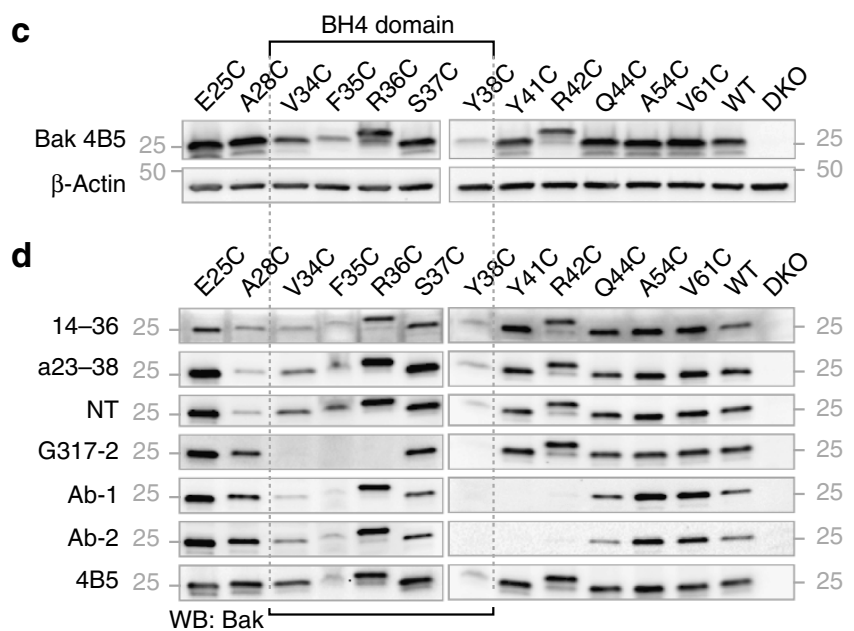

Figure 2 | Epitopes in $\boldsymbol{\alpha} \mathbf{1}$ and residues of $\boldsymbol{\alpha} \mathbf{1}$ important for Bak stability. (a) Precise positioning of the G317-2, Ab-1, Ab-2 and 7D10 epitopes. Histograms showing immunoreactivity of Bak antibodies towards biotinylated 8-mer peptides that collectively span residues 20-65 of human Bak. X axis labels indicate peptide sequence or control reaction conditions. Data are mean and s.d. of three independent experiments. (b) Diagrams showing the position of the BH4 domain ${ }^{15}$ in the Bak $\alpha 1$ sequence and structure of inactive Bak (PDB: 2 IMS, ref. 3 ). Residues in bold were mutagenized for experiments illustrated in (c) and (d). Three different orientations of the structure are shown. Note the hydrophobic side-chains of the BH4 domain (red) within $\alpha 1$ (yellow) point towards $\alpha 5$ (pink) and $\alpha 6$ (cyan) residues in the core of Bak. (c) Hydrophobic residues in the BH4 domain are important for Bak function. Lysates from DKO MEF expressing Bak $\alpha 1$ cysteine mutants were analysed by western blot. Y38C, F35C and, to a lesser extent, V34C exhibited consistently weaker signals than other $\alpha 1$ mutants. E25C migrates a little faster and R36C and R42C slightly slower than WT hBak, due to their cysteine substitutions changing the overall net charge of $\mathrm{Bak}^{40}$. Blots were re-probed with $\beta$-actin to compare loading. Data are representative of two independent experiments. (d) Three sets of Bak $\alpha 1$ residues (A28, V34-R36 and S37-Q44) are important for antibody binding. The ability of antibodies (indicated on the left) to bind Bak $\alpha 1$ cysteine mutants (indicated above) was compared by western blotting. Lysates from DKO MEF expressing no Bak, WT Bak or mutations C-terminal to $\alpha 1$ (A54C, V61C) were included as negative and positive controls. Data are representative of three independent experiments.

the A28C:L163C tether (Supplementary Fig. 4). Tethering of WT Bak had an intermediate effect on BH4 exposure, as both G317-2 and $\mathrm{Ab}-1$ binding occurred in the majority of Bid-treated cells (Fig. 5a,b,d,e), but less antibody bound to each cell (Fig. 5c,f). We interpret the lower mean fluorescence intensity (MFI) to indicate that all Bak molecules changed conformation in response to Bid, but were only partially exposing the G317-2 and Ab-1 epitopes. Thus, the WT tether is less restrictive than the A28C:L163C tether, and allows sufficient $\alpha 1$ dissociation for some antibody to bind. Notably, when Bak was activated by a Bim BH3 peptide or by heating, $\mathrm{BH} 4$ exposure was again restricted by the WT and A28C:L163C tethers (Supplementary Fig. 5), suggesting that $\alpha 1$ dissociation from $\alpha 6$ is a requisite response to all triggers of Bak activation.

Full BH4 exposure requires core-latch dissociation. Recent studies show that activation of both Bak and Bax requires separation of $\alpha 5$ in the core from $\alpha 6$ in the latch ${ }^{19,20}$, raising the possibility that this event occurs before $\alpha 1$ dissociation. For example, the $\alpha 1-\alpha 6$ tether in Fig. 5 might be preventing $\alpha 6$ dissociation rather than $\alpha 1$ dissociation. To test the relationship of core-latch separation to $\alpha 1$ dissociation, we utilized the Bak variant V142C/F150C (ref. 20). When treated with CuPhe,
V142C in $\alpha 5$ was efficiently tethered to F150C in $\alpha 6$ and the tether prevented cytochrome $c$ release in response to $\mathrm{Bid}^{20}$. We now show that when $\mathrm{V} 142 \mathrm{C} / \mathrm{F} 150 \mathrm{C}$ is tethered, binding of both G317-2 and Ab-1 still occurs in the majority of Bid-treated cells (Fig. 6a, b), as found for tethered WT Bak (Fig. 5). As binding of $\mathrm{Ab}-1$ would not occur after dissociation of $\alpha 6-\alpha 8$ alone (Supplementary Fig. 3), $\alpha 1$ dissociation must be initiated before or in concert with core-latch separation. In addition, as antibody binding is limited in the presence of the tether (Fig. 6c), complete $\alpha 1$ dissociation requires $\alpha 6$ dissociation from the core. Altogether, these results indicate that movement of both $\alpha 1$ and the a6-containing latch are required for full exposure of the $\mathrm{BH} 4$ domain during activation.

In addition, we found that another measure of Bak $\alpha 1$ conformation change was cleavage by the serine protease enterokinase (EK). EK cleaved Bak only after it had been activated by Bid, and generated an $\sim 20 \mathrm{kDa}$ membrane-bound fragment (Supplementary Fig. 6). Moreover, the non-canonical cleavage site was within the $\mathrm{BH} 4$ domain in $\alpha 1$ as the cleaved fragment was recognized by Ab-1 but not G317-2 (Fig. 7a). Notably, cleavage was absent when the $\mathrm{N}$ terminus was tethered to the latch (WT, A28CL163C) or the latch was tethered to the core (V142CF150C, Fig. 7b), indicating that those tethers had blocked BH4 exposure, consistent with our FACS results. 
a Endogenous mouse Bak $\left(\mathrm{Bax}^{-/-} \mathrm{MEF}\right)$

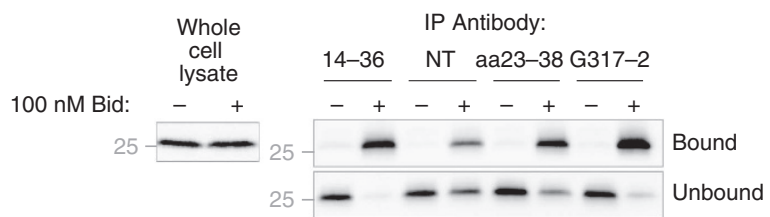

b Endogenous human Bak (DU145)

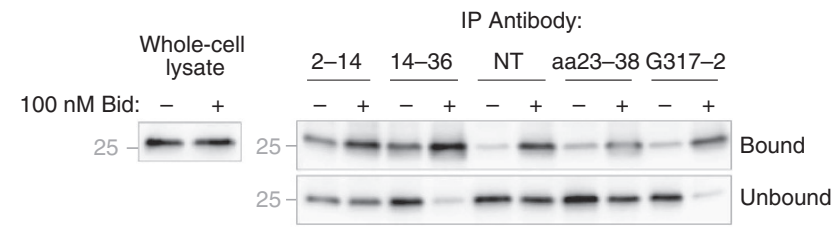

C Exogenous human Bak (DKO MEF+WT hBak)

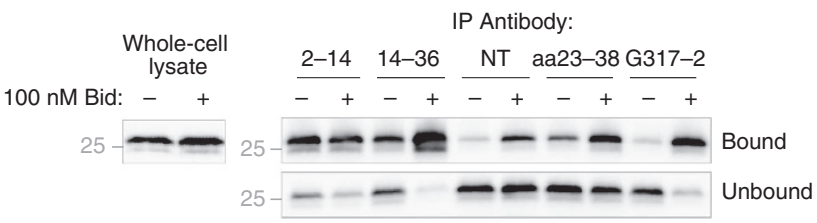

Figure 3 | All Bak N-terminal epitopes are exposed during activation.

Membrane fractions from cells expressing endogenous mouse or human Bak (a,b respectively) or exogenous human Bak (c) were incubated (30 min, $30^{\circ} \mathrm{C}$ ) with $(+)$ or without $(-)$ Bid, solubilized with digitonin, and incubated with the Bak antibodies shown. Bak in immunoprecipitates and whole-cell lysate or unbound controls was detected by western blot using the 4B5 antibody. Data are representative of at least two independent experiments.

Curiously, cleavage was also absent when $\alpha 1$ was tethered to $\alpha 2$ (Y41CA79C, Fig. 7b) despite full exposure of the G317-2 epitope (Figs $4 \mathrm{~d}$,e and $5 \mathrm{a}-\mathrm{c}$ ). We suspect the disulfide bond prevents access of EK, but not G317-2, to its target site. These results therefore confirm that movement of both $\alpha 1$ - and the a6-containing latch are required for full $\mathrm{BH} 4$ exposure during Bak activation.

BH3 exposure is initiated in the absence of $\mathrm{BH} 4$ exposure. To test whether $\alpha 2$ movement might precede $\alpha 1$ movement, we measured $\mathrm{BH} 3$ exposure when $\alpha 1$ was tethered or untethered. It was plausible that $\mathrm{BH} 3$ exposure could occur even if $\alpha 1$ was tethered to the protein core due to the length (19 residues) and flexibility of the $\alpha 1-\alpha 2$ loop. To capture BH3 exposure, the 4B5 antibody was added during (rather than after) the incubation with $\mathrm{Bid}$, as the $\mathrm{BH} 3$ domain is normally only transiently exposed before it binds to another activated Bak molecule 9 . Accordingly, in the untethered samples, 4B5 added during the incubation bound each Bak variant after Bid treatment (Fig. 8a,b). Even in the tethered samples, the $4 \mathrm{~B} 5$ antibody bound to all three Bak variants (Fig. 8a,b) indicating that $\mathrm{BH} 3$ exposure is a very early step in Bak activation. The $\alpha 1-\alpha 6$ tether (A28C:L163C) was most informative as it fully blocked $\mathrm{BH} 4$ exposure (Fig. 5) but not $\mathrm{BH} 3$ exposure (Fig. 8a,b). Thus, when Bid binds to the Bak hydrophobic groove, $\mathrm{BH} 3$ exposure is initiated before $\mathrm{BH} 4$ exposure.

We also noted that $4 \mathrm{~B} 5$ binding was limited in tethered WT and $\mathrm{A} 28 \mathrm{C} / \mathrm{L} 163 \mathrm{C}$, as shown by lower MFI after tethering (Fig. 8c). This limited 4B5 binding suggests that flexion of the $\alpha 1-\alpha 2$ loop is not sufficient for complete BH3 exposure. Instead, $\alpha 1$ dissociation from the $\alpha 6-\alpha 8$ latch domain is also required, as suggested by complete BH3 exposure when $\alpha 1$ was tethered to $\alpha 2$ (Y41C:A79C, Fig. 8a-c).

Dimer formation requires dissociation of $\boldsymbol{\alpha} \mathbf{1}$ from $\boldsymbol{\alpha} \mathbf{2}$. We noted that one tether (Y41C:A79C) allowed Bid treatment to expose both the $\mathrm{BH} 4$ and $\mathrm{BH} 3$ domains (Figs 5a,b and 7a,b) but did not allow cytochrome $c$ release (Fig. 4c). As $\alpha 1$ was still linked to $\alpha 2$, the exposed $\mathrm{BH} 3$ domain may have been unable to bind the hydrophobic groove of another activated Bak molecule to form a BH3:groove homodimer. This was confirmed by Blue-NativePAGE experiments in which tethered Bak Y41C/A79C failed to dimerize following Bid treatment (Fig. 8d). In addition, the $\mathrm{BH} 3$ domain remained exposed as shown by $4 \mathrm{~B} 5$ antibody binding after (rather than during) the Bid incubation (Fig. 8e,f). Thus, Bak $\alpha 1$ must dissociate from $\alpha 2$ for Bak dimerization and pore formation.

In summary, Bak activation by Bid, Bim or heat proceeds via movement of $\alpha 2$ followed by dissociation of $\alpha 1$ from both the $\alpha 2-\alpha 5$ core domain and the $\alpha 6-\alpha 8$ latch domain. Separation of the latch from core does not occur before $\alpha 1$ dissociation, but may occur in concert as contacts between helices are progressively lost. The resulting exposure of hydrophobic residues in multiple helices may drive further conformation changes that promote Bak oligomerization due to the newly exposed residues engaging in new protein-protein or protein-lipid interactions.

\section{Discussion}

To delineate the Bak 'N-terminal conformation change' known for many years to occur during apoptosis, we mapped N-terminal epitopes (summarized in Fig. 9a) and assayed their exposure in combination with disulfide tethering (Figs 4-8). Our data show that during apoptosis, the $\mathrm{N}$-segment and $\alpha 1$ of Bak dissociate entirely from the rest of the protein to expose the $\mathrm{BH} 4$ domain and other residues (Fig. 9b), consistent with recent reports that Bak $\alpha 1$ and $\alpha 6$ became $>50 \AA$ apart after tBid treatment in $\mathrm{LUV}^{26}$ and the distance between Bax $\alpha 1$ and $\alpha 2$ varies widely after activation ${ }^{21}$. Disulfide tethering of Bak showed that $\alpha 1$ dissociation from both the core $(\alpha 2-\alpha 5)$ and the latch $(\alpha 6-\alpha 8)$ was required for pore formation. Furthermore, our novel approach of combining tethers with FACS analysis of epitope exposure suggested that Bak conformation change initiates with movement of $\alpha 2$, followed by $\alpha 1$ dissociation, and that both events are required for complete $\mathrm{BH} 3$ exposure and subsequent dimerization.

The requirement of $\alpha 1$ dissociation for Bak function is consistent with the role of $\alpha 1$, and more specifically the BH4 domain, in stabilizing the non-activated conformation of Bak. The BH4 motif is highly conserved in multidomain Bcl-2 proteins including viral prosurvival proteins ${ }^{15}$. Its role in stabilizing Bak is indicated by the structure of non-activated Bak in which the four hydrophobic residues in the $\mathrm{BH} 4$ domain $\left({ }^{34} \mathrm{VFxxYV}^{39}\right)$ contact four neighbouring helices $(\alpha 2, \alpha 5, \alpha 6, \alpha 7)^{3}$. R42 may also stabilize non-activated Bak, as structurally this residue participates in a hydrogen bond network with $\alpha 3$ (ref. 3).

As $\alpha 1$ makes many contacts with other helices, $\alpha 1$ dissociation may be triggered by disrupting one or more of those contacts. A recent crystal structure of Bim peptide bound to the Bax groove demonstrated $\alpha 2$ movement, and formation of a cavity between helices 2, 5 and 8 (ref. 19). An NMR structure of Bid peptide bound to the Bak groove also indicated perturbations in several helices including $\alpha 1, \alpha 2$ and $\alpha 6$ (ref. 12). The present studies show $\alpha 2$ movement as a very early consequence of Bid binding to the Bak groove. Thus, $\alpha 2 / 3$ movement and cavity formation may individually or in concert trigger $\alpha 1$ dissociation. 
a

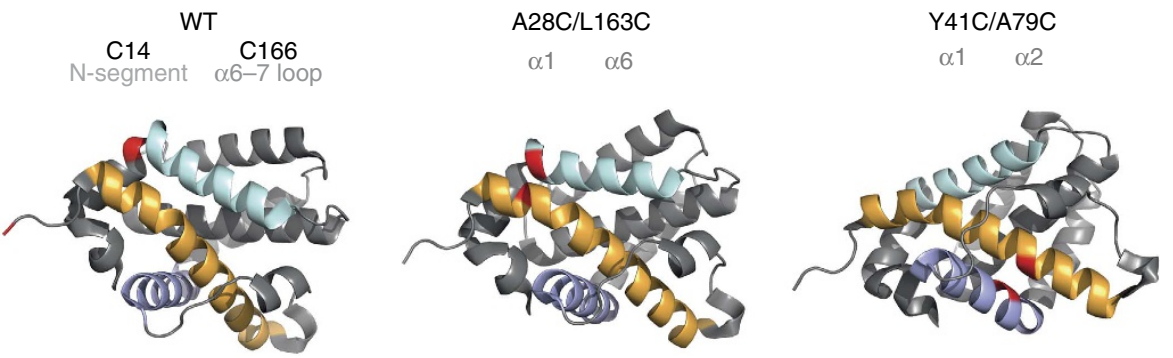

b

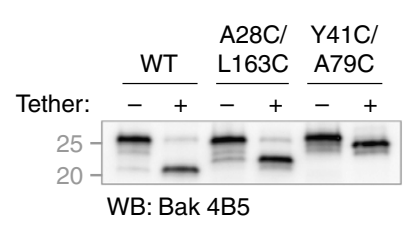

C
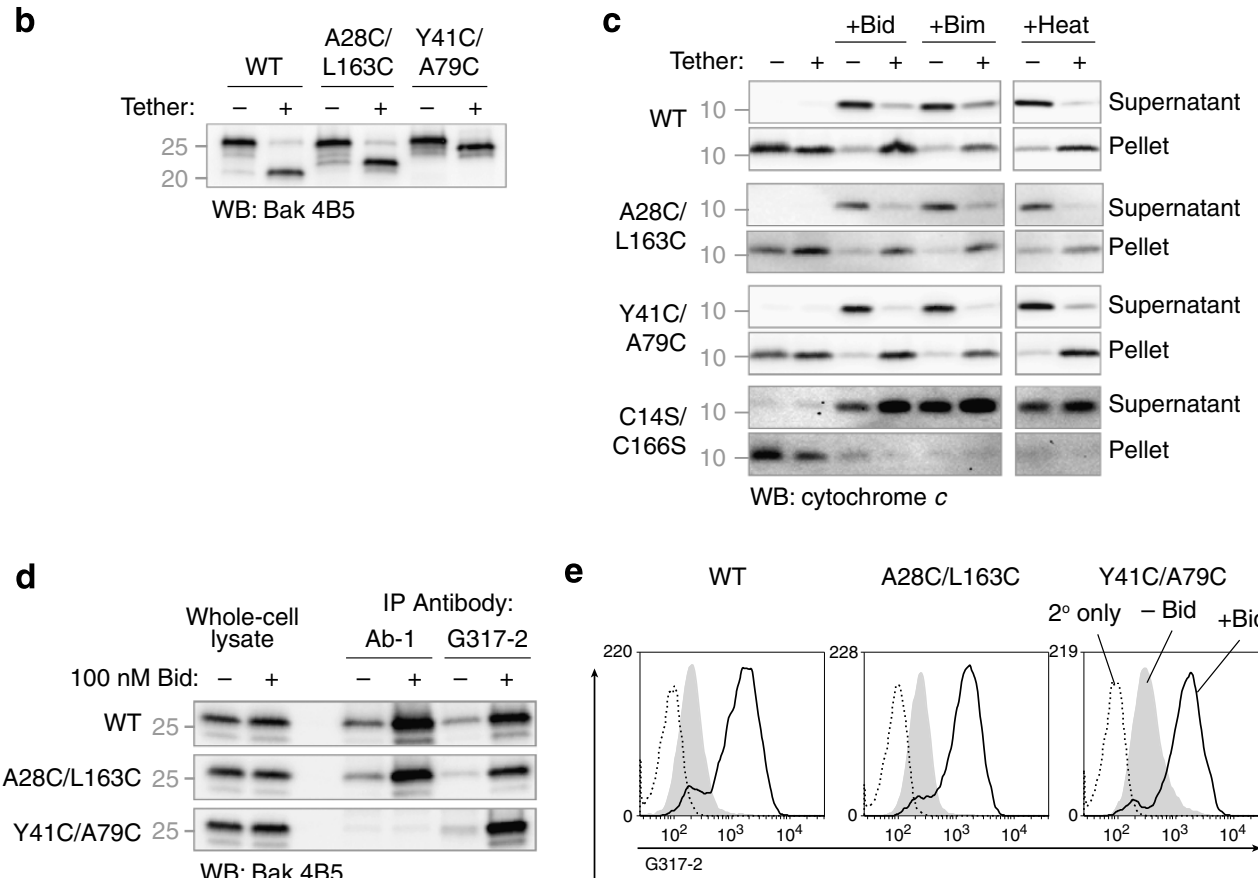

e
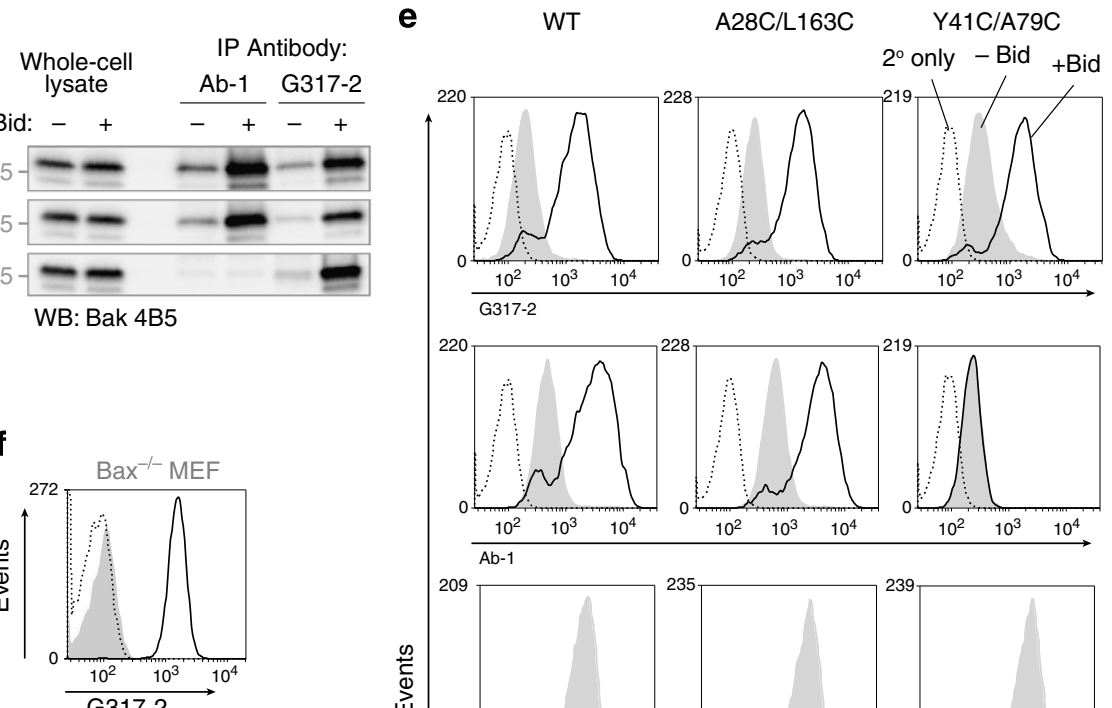

岂
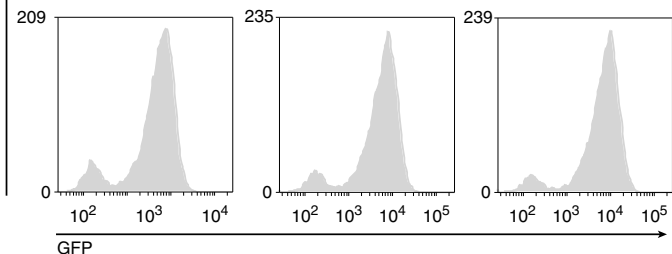

Figure 4 | N-terminal tethers and G317-2 detection of Bak activation by intracellular FACS. (a) Position of residues (red) in the inactive Bak structure (PDB: 2IMS, ref. 3) that can be cross-linked with CuPhe to generate tethers; $\alpha 1$ is yellow, $\alpha 6$ is cyan and $\alpha 2$ is lilac. As the structure was derived using truncated $\mathrm{Bak}^{3}$, for WT Bak the most N-terminal residue present (S21) is marked red as a surrogate for C14. (b) Tethers were efficiently induced by CuPhe cross-linking of cysteines in non-activated Bak. DKO MEF were incubated with $(+)$ or without $(-) 200 \mu \mathrm{M} \mathrm{CuPhe} \mathrm{for} \mathrm{at} \mathrm{least} 5$ min, on ice. Tethered Bak appears as a faster migrating fragment after non-reducing SDS-PAGE. Differences in migration between samples reflect the positions of the tethers. Data are representative of at least three independent experiments. (c) Bak mutants are functional and all tethers block cytochrome $c$ release in response to apoptotic stimuli. After inducing tethers (as in b), membrane fractions were incubated with or without either $100 \mathrm{nM} \mathrm{Bid,} 10 \mu \mathrm{M}$ Bim peptide or heat $\left(44^{\circ} \mathrm{C}\right.$ ). Data are representative of three independent experiments. (d) G317-2, but not Ab-1, can bind to activated Y41CA79C Bak. Membrane fractions from DKO MEF expressing hBak were treated as in Fig. 3. Data are representative of three independent experiments. (e) G317-2 can substitute for Ab-1 in intracellular FACS assays for Bak activation. Permeabilized DKO MEF were treated with (solid line) or without (filled histograms) $100 \mathrm{nM}$ Bid and incubated with Ab-1 or G317-2. Dotted lines show signals for cells incubated without primary antibody. FACS plots of GFP levels indicate the proportion of cells expressing Bak. The G317-2 profiles of treated cells closely match GFP levels, indicating all cells expressing Bak responded to Bid. (f) Mouse Bak activation can be measured by intracellular FACS using G317-2. Bax-1- MEF were treated as in (e). 

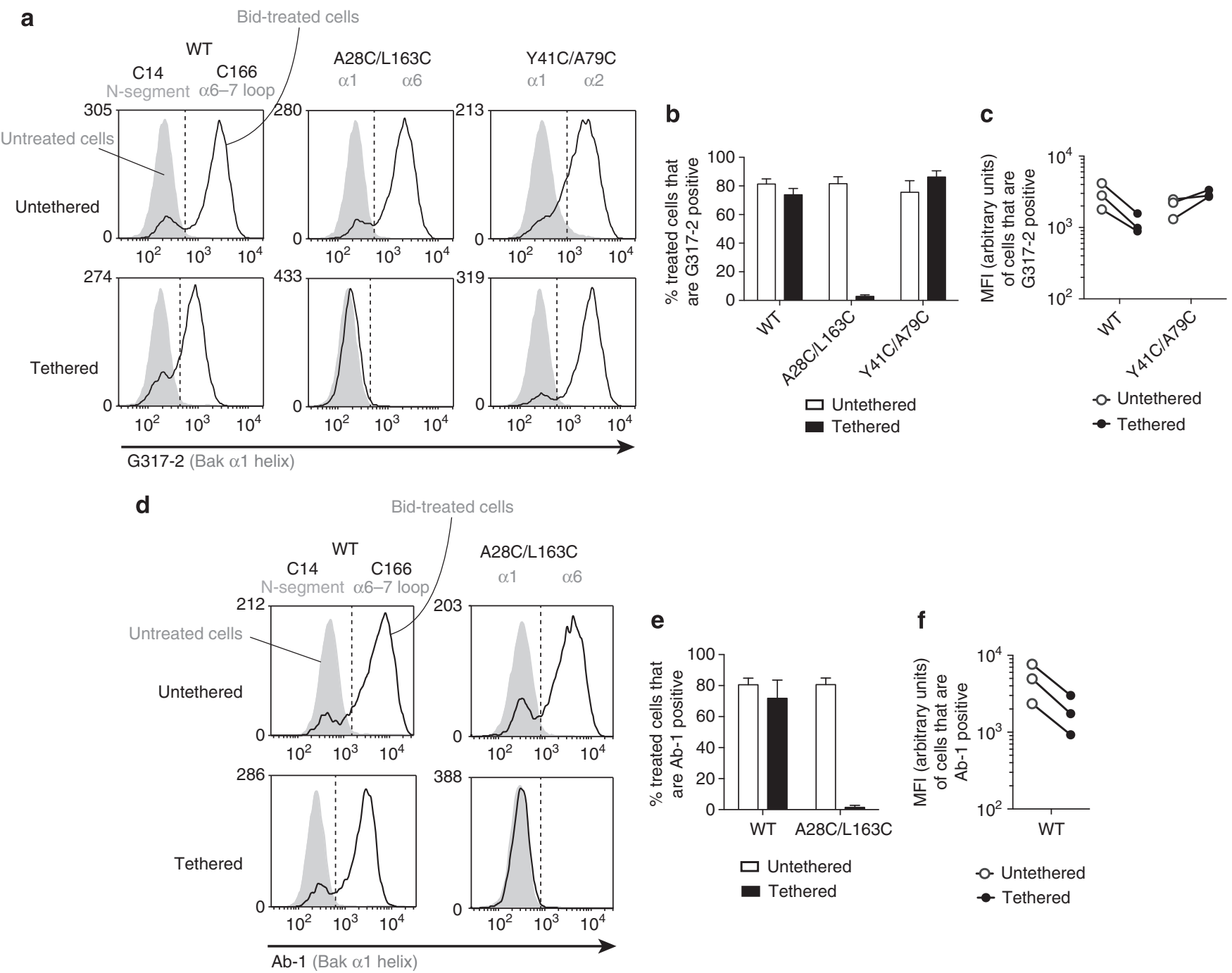

Figure 5 | Bak BH4 exposure requires dissociation of $\boldsymbol{\alpha} \mathbf{1}$ from $\alpha \mathbf{6}$. (a) Tethering the $\alpha 1$ and $\alpha 6$ helices completely blocks BH4 exposure. Permeabilized DKO MEF expressing Bak (as indicated) were incubated with (tethered) or without (untethered) $200 \mu \mathrm{M}$ CuPhe, then treated for 30 min with (black lines) or without (filled histograms) $100 \mathrm{nM} \mathrm{Bid}\left(30^{\circ} \mathrm{C}\right)$. BH4 exposure was determined by staining with G317-2. Representative FACS plots are shown.

(b) Proportion of cells with exposed G317-2 epitope after Bid treatment. The graph shows the percentage cells right of the vertical lines in FACS plots, in Bid-treated samples. Data in graph are mean and s.d. of at least three independent FACS experiments including the data shown in a. (c) Tethering the $\mathrm{N}$-segment to the $\alpha 6-\alpha 7$ loop prevents complete G317-2 exposure. Comparison of the mean fluorescent intensities (MFI) of the WT and Y41C/A79C cell populations graphed in $\mathbf{b}$. Lines connect data from the same experiment. (d) Tethering the $N$ terminus to the latch blocks any movement of $\alpha 1$ in response to Bid. Permeabilized DKO MEF expressing WT or A28C/L163C Bak were treated as in a and exposure of an $\alpha 1$ epitope (Ab-1) more distant to the tether than G317-2 (Supplementary Fig. 4) was assessed by FACS. (e) Proportion of cells with exposed Ab-1 epitope after Bid treatment. The graph shows the percentage cells right of the vertical lines in FACS plots, in Bid-treated samples. Data in graph are mean and s.d. of at least three independent FACS experiments including the data shown in $\mathbf{d}$. (f) Tethering the $\mathrm{N}$-segment to the $\alpha 6-\alpha 7$ loop prevents complete Ab- 1 exposure. Comparison of the mean fluorescent intensities (MFI) of WT cell populations graphed in e. Lines connect data from the same experiment.

Dissociation of $\alpha 1$ allows Bak to unfold into three major components: the $\mathrm{N}$ terminus, the core $(\alpha 2-\alpha 5)$ and the latch $(\alpha 6-\alpha 8)$. This unfolding exposes many hydrophobic residues in $\alpha 1, \alpha 2$ and $\alpha 5-\alpha 8$ (Supplementary Fig. 7) that engage in new protein-lipid or protein-protein interactions to porate the MOM. For example, recent structural and biochemical studies indicate that protein-lipid interactions occur as Bak collapses onto the membrane, with $\alpha 4, \alpha 5$ and $\alpha 6$ lying in-plane on the MOM surface $^{19,26}$. The amphipathic $\alpha 7$ and $\alpha 8$ may also lie in-plane to protect their hydrophobic and aromatic residues. Protein-protein interaction generates dimers when the exposed $\mathrm{BH} 3$ domain in $\alpha 2$ binds to the hydrophobic groove of another Bak molecule ${ }^{9,22}$. In contrast, $\alpha 1$ remains solvent-exposed as all epitopes can be bound by antibodies in immunoprecipitation or intracellular
FACS assays, including those that involve hydrophobic regions such as the BH4 domain. Solvent-exposure of the $\mathrm{N}$ terminus is consistent with increased susceptibility of activated Bak to proteolysis by proteinase $\mathrm{K}$, trypsin, calpain ${ }^{11,27-29}$ and enterokinase (Fig. 7). Thus, unlike the core and latch domains, the $\mathrm{N}$ terminus does not participate in forming the apoptotic pore, consistent with the ability of $\mathrm{N}$-terminally truncated Bak or Bax to permeabilize mitochondria ${ }^{14,30}$.

Dissociation of $\alpha 1$ may also be necessary for heterodimerization with the prosurvival proteins $\mathrm{Bcl}-\mathrm{x}_{\mathrm{L}}$ and $\mathrm{Mcl}-1$, as heterodimers form via a $\mathrm{BH} 3$ :groove interaction ${ }^{27,31}$. Dissociation of $\alpha 1$ before heterodimerization is consistent with exposure of a calpain cleavage site at R42 in $\alpha 1$ in the Bak:Bcl- $\mathrm{x}_{\mathrm{L}}$ complex $^{27}$, and even with interaction of the Bax $\alpha 1$ with $\alpha 1$ of $B c l-x_{L}$ (ref. 32). 

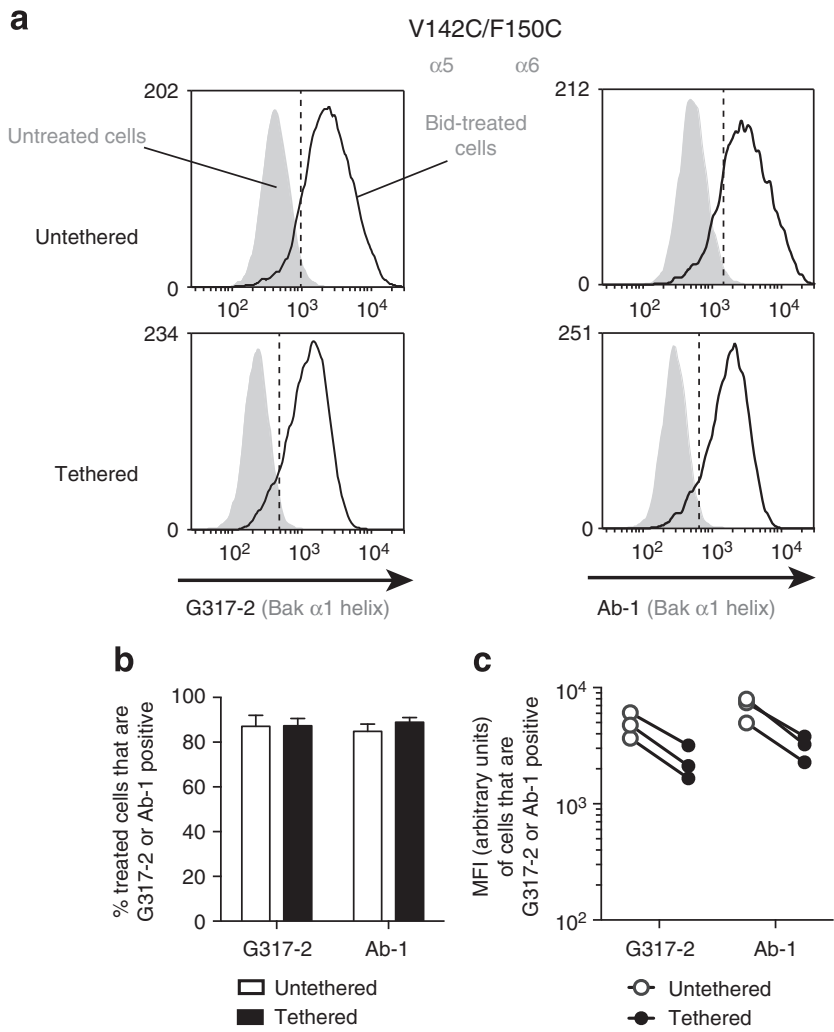

Figure 6 | Full BH4 exposure requires core-latch dissociation.

(a) Dissociation of $\alpha 1$ occurs before or in concert with $\alpha 6$ movement. Permeabilized DKO MEF expressing V142C/F150C Bak were treated with CuPhe and Bid as in Fig. 5a. Dissociation of $\alpha 1$ was measured by staining with G317-2 and Ab-1. Representative FACS plots are shown. (b) Proportion of cells with exposed $\mathrm{BH} 4$ domain in response to Bid. The graph shows the percentage of cells right of the vertical lines in FACS plots, in Bid-treated samples. Data are mean and s.d. of at least three independent FACS experiments, including the data shown in $\mathbf{a}$. (c) Tethering the core and latch prevents complete $\mathrm{BH} 4$ exposure. Comparison of the mean fluorescent intensities (MFI) of V142/CF150C cell populations graphed in $\mathbf{b}$. Lines connect data from the same experiment.

Where $\mathrm{Bcl}-\mathrm{x}_{\mathrm{L}}$ has apparently prevented exposure of N-terminal epitopes in Bak or Bax (as measured by immunoprecipitation assays) ${ }^{17,25,27}$, it is likely that $\mathrm{Bcl}-\mathrm{x}_{\mathrm{L}}$ prevented Bak/Bax activation via Mode 1 interactions with $\mathrm{BH} 3$-only proteins $\mathrm{s}^{27}$ or that it masked the Bak or Bax epitopes.

Three factors hint that $\alpha 1$ may not retain the helical conformation evident in the structure of non-activated $\mathrm{Bak}^{3}$. First, all epitopes in $\alpha 1$ appear linear based on their binding to Bak after SDS-PAGE and to the peptide array. Second, calpain cleaves activated Bak at R42/H43 toward the end of $\alpha 1$ (ref. 12), and calpain rarely cleaves in helical regions ${ }^{33}$. Third, if $\alpha 1$ did remain helical, the hydrophobic surface (for example, the $\mathrm{BH} 4$ domain) that normally faces the hydrophobic core of Bak might be expected to make further protein-protein or protein-lipid interactions. But instead, all regions remain available for antibody binding.

Like Bak, Bax N-terminal epitopes (for the 6A7, Bax NT and Bax N20 antibodies) become exposed following apoptosis. Moreover, the epitopes are in the region of $\operatorname{Bax} \alpha 1$ (residues $13-19,1-21$ and 11-30, respectively) that abuts the $\alpha 6-\alpha 8$ latch domain $^{34-36}$, and the epitopes of polyclonal Bax N20 may even include residues of the Bax BH4 domain $\left({ }^{26} \mathrm{LLQGFI}^{31}\right)$. As Bax $\mathrm{N}$-terminal exposure is akin to that for Bak, $\alpha 1$ dissociation is probably also required for Bax activation and pore formation. a

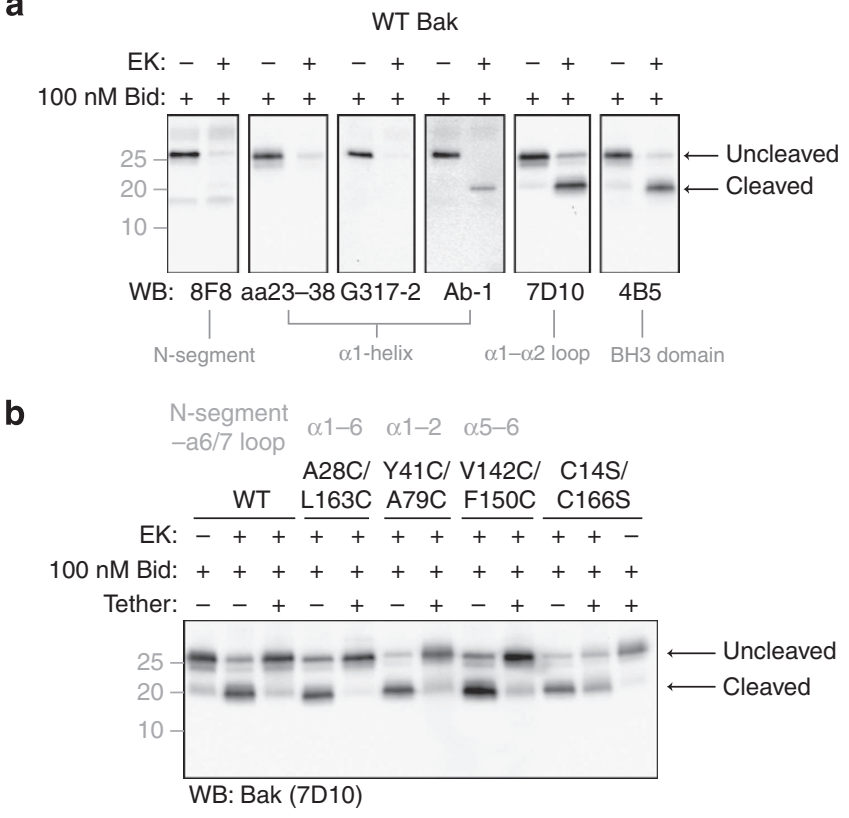

Figure 7 | Tethering Bak prevents exposure of an enterokinase cleavage site in the BH4 domain. (a) Enterokinase cleaves at the $\mathrm{BH} 4$ domain in activated Bak. Permeabilized DKO MEF expressing WT Bak were treated with Bid $(+)$ as in Fig. $5 \mathrm{a}$, then incubated for $2 \mathrm{~h}$ at room temperature without $(-)$ or with recombinant enterokinase (EK; 3 U per $50 \mu l)$. Samples were western blotted with the indicated anti-Bak antibodies. Note that the BH4 domain spans the G317-2 and Ab-1 epitopes (see Fig. 9a). Data are representative of at least two independent experiments. (b) Tethering Bak prevents exposure of enterokinase cleavage site. Permeabilized DKO MEF expressing the indicated Bak variants were treated with CuPhe and Bid as in Fig. $5 \mathrm{a}$ and with EK as in Fig. 7a and then western blotted with the 7D10 antibody as Ab-1 does not bind Y41C/A79C Bak. Note EK was still able to cleave Bid-treated C14S/C166S Bak in the presence of CuPhe. Data are representative of at least two independent experiments.

Indeed, tethering the Bax $\alpha 1-\alpha 2$ loop to $\alpha 6$ blocked $6 \mathrm{~A} 7$ exposure and cytochrome $c$ release in response to SAHB Bim peptide ${ }^{37}$. In this case, the binding site was the 'rear pocket' formed by Bax $\alpha 1 / \alpha 6$, suggesting that $\alpha 1$ dissociation is a key event in Bax and Bak activation regardless of the initiating interaction.

In conclusion, detailed characterization of the epitopes of several commonly used Bak antibodies, together with a novel tethering approach, enabled us to determine that $\alpha 1$ dissociation is a crucial step in Bak (and probably Bax) activation. With the restraining influence of $\alpha 1$ removed, the freed core and latch domains are able to orient in the membrane, enabling dimerization, oligomerization, cytochrome $c$ release and ultimately cell destruction. Thus, interference with $\alpha 2$ movement or $\alpha 1$ dissociation may represent a new strategy for therapeutic intervention in pathologies associated with overactive or inefficient Bak-mediated apoptosis.

\section{Methods}

Antibodies and other materials. Anti-Bak antibodies were obtained from suppliers and diluted as indicated in Table 1. Other primary antibodies used were anticytochrome $c$ (Clone 7H8.2C12, 1:1,000, BD \#556433) and anti $\beta$-actin (Clone AC15, 1:20,000, Sigma \#A1978). Secondary antibodies used for western blotting were horseradish peroxidase (HRP)-conjugated sheep anti-mouse (1:2,000, Amersham \#NXA931), goat anti-rabbit (1:5,000, Southern Biotech \#4010-05) and goat anti-rat (1:5,000, Southern Biotech \#3010-05) IgGs. To avoid nonspecific signals from the light chains of IP antibodies, which are very similar in size to Bak, $\mathrm{FC}_{\Upsilon}$ chainspecific HRP-conjugated goat anti-rat IgG (1:5,000, Southern Biotech \#3030-05) was used for IP western blots (Figs 3 and 4d). Secondary antibodies used for FACS 

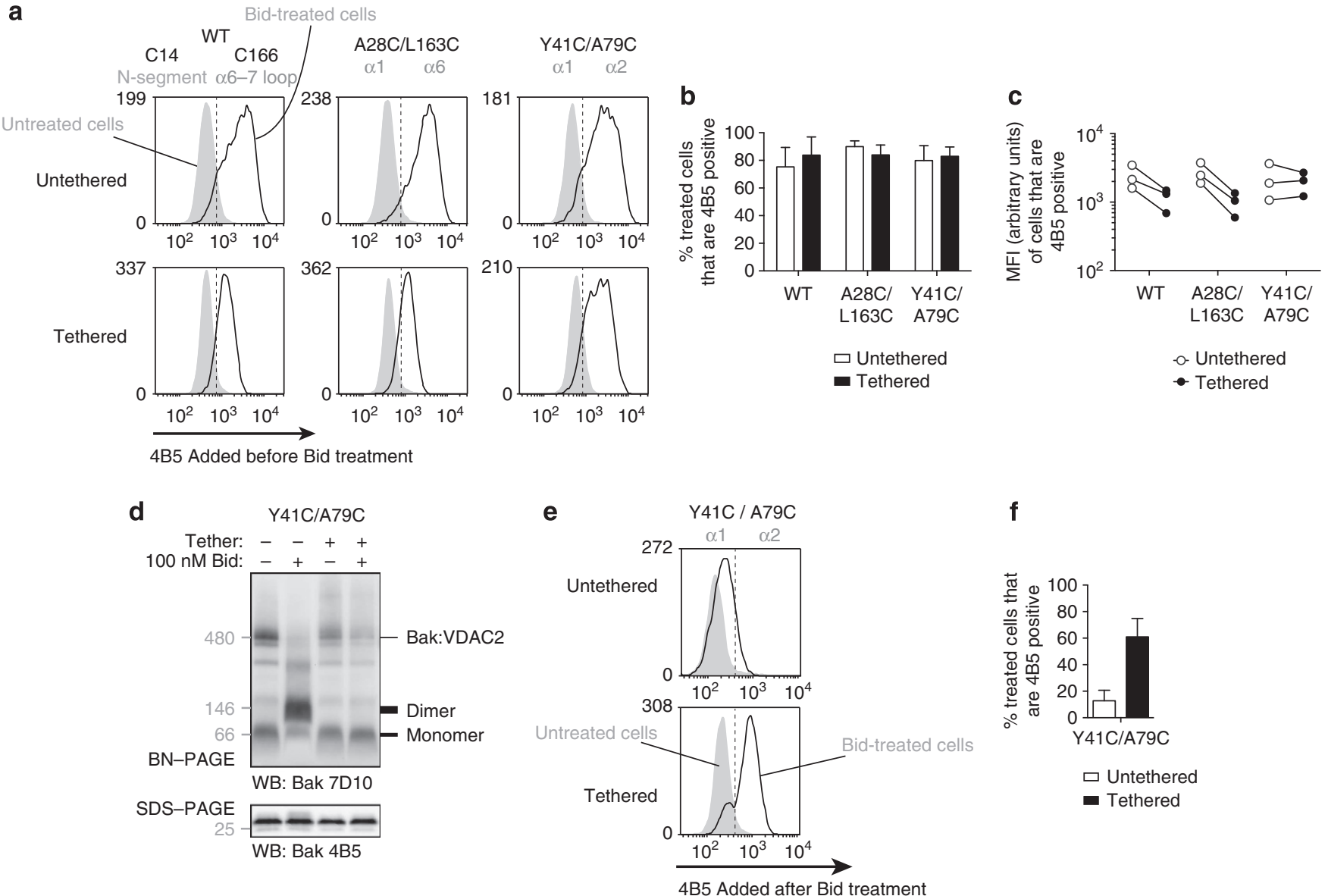

Figure 8 | Bak BH3 exposure precedes BH4 exposure, but dimerization requires dissociation of $\boldsymbol{\alpha 1}$ from $\boldsymbol{\alpha 2}$. (a) Tethering of the $\mathrm{N}$ terminus to $\alpha 2$ or the 66- $\alpha 8$ latch allows BH3 exposure. Permeabilized DKO MEF expressing Bak (as indicated) were treated with CuPhe and Bid as in Fig. 5a. BH3 exposure was detected by the addition of $4 \mathrm{~B} 5$ to cells before Bid, because the $4 \mathrm{~B} 5$ epitope is part of the BH3:groove interface ${ }^{9}$ and thus inaccessible after Bid treatment. Representative FACS plots are shown. (b) Proportion of cells with an exposed BH3 domain in response to Bid. The graph shows the percentage of cells, right of the vertical lines in the plots, in Bid-treated samples. Data are mean and s.d. of at least three independent FACS experiments, including the data shown in a. (c) The $\mathrm{N}$-segment and $\alpha 1$ must dissociate from the $\alpha 6-\alpha 8$ latch for complete BH3 exposure. Comparison of mean fluorescent intensities (MFIs) of cell populations graphed in $\mathbf{b}$. Lines connect data from the same experiment. (d) Tethering $\alpha 1$ to $\alpha 2$ prevents BH3:groove dimerization. Permeabilized DKO MEF expressing Y41C/A79C Bak were treated with CuPhe and Bid as in Fig. $5 \mathrm{a}$. Bak dimers and monomers were resolved by Blue Native (BN)-PAGE and visualized after western blot. SDS-PAGE of samples indicated equivalent loading. Data are representative of two independent experiments. (e) Tethering $\alpha 1$ to $\alpha 2$ results in persistent exposure of $\mathrm{BH} 3$ domains in response to Bid. Permeabilized DKO MEF expressing Y41C/A79C Bak were treated with CuPhe and Bid as in Fig. $5 \mathrm{a}$ and subsequently incubated with 4B5. Representative FACS plots are shown. (f) Proportion of cells with BH3 domains exposed after responding to Bid, indicating an inability to form homodimers. The graph shows the percentage of cells right of the vertical line, after Bid treatment. Data in graph are mean and s.d. of at least three independent FACS experiments, including the data shown in $\mathbf{e}$.

were RPE-labelled goat anti-rat (1:200, Southern Biotech \#3050-09) or goat antimouse (1:200, Southern Biotech \#1031-09) IgGs.

Bim BH3 peptide ${ }^{38}$ (H-DMRPEIWIAQELRRIGDEFNAYYARR-NH2) was synthesized by Mimotopes, reconstituted in dimethyl sulfoxide $(10 \mathrm{mM})$ and kept at $-20^{\circ} \mathrm{C}$.

Redox catalyst copper(II)(1,10-phenoanthroline) ${ }_{3}$ (CuPhe) stocks were prepared with $30 \mathrm{mM} \mathrm{CuSO}_{4}$ and $100 \mathrm{mM}$ 1,10-phenoanthroline in 4:1 water:ethanol and stored at $-20^{\circ} \mathrm{C}$

Cell culture. Phoenix cells, DU145 and SV40-transformed mouse embryonic fibroblasts derived from $B a k^{-/-} \mathrm{Bax}^{-1-}$ mice (DKO MEF) and $\mathrm{Bax}-1-$ mice were a gift from Professor David Huang. Note, the DU145 cells were obtained from the Frederick National Laboratory (USA) as part of the NCI-60 panel of cell lines ${ }^{39}$.

DKO MEF, DKO MEF expressing Bak variants (see below), $\mathrm{Bax}^{-/-} \mathrm{MEF}$ and Phoenix cells were maintained in Dulbecco's modified Eagle's medium (DMEM) supplemented with $10 \%$ fetal calf serum (FCS), $250 \mu \mathrm{M}$ L-asparagine and $55 \mu \mathrm{M}$ 2-mercaptoethanol. DU145 cells were maintained in DMEM supplemented with $10 \%$ FCS. All lines were routinely passaged by rinsing cells with phosphatebuffered saline (PBS) and incubating with trypsin for 3-10 min, and then resuspending cells (diluted 1:2 to $1: 30$ ) in fresh medium. All lines were cultured at $37^{\circ} \mathrm{C}$ in $10 \% \mathrm{CO}_{2}$.
Site-directed mutagenesis. Cysteine variants and N-terminal truncations of Bak were generated by cloning the product of three sequential PCRs (see below) into the pMX-IG vector, kindly provided by Professor David Huang. Primers (Supplementary Table 1) were purchased from GeneWorks Pty Ltd.

For each mutation (except the truncations), two initial paired reactions were performed. One reaction contained the relevant internal (mutagenic) forward primer and a Bak reverse primer that includes an XhoI site. The second reaction contained an hBak forward primer that includes an EcoRI site and the matching internal (mutagenic) reverse primer. Each reaction mixture contained $20 \mathrm{ng}$ template DNA, $0.5 \mu \mathrm{M}$ of each primer and $1 \times$ Phusion PCR Master Mix (Finnzymes, \#F-531S) and were incubated in an MJ Research PTC-200 Thermal Cycler or a Bio-Rad T100 Thermal Cycler. Cycling conditions were: $94^{\circ} \mathrm{C}, 2 \mathrm{~min}$; 30 cycles of $\left(94^{\circ} \mathrm{C}, 35 \mathrm{~s} ; 58^{\circ} \mathrm{C}, 30 \mathrm{~s} ; 72^{\circ} \mathrm{C}, 60 \mathrm{~s}\right) ; 72^{\circ} \mathrm{C}, 5 \mathrm{~min}$.

The two resulting complementary DNA fragments were gel-purified using the Wizard SV Gel and PCR Cleanup kit (Promega, \#A9282) and each pair of DNAs ( $3 \mu \mathrm{l}$ each) annealed by incubation with $1 \times$ Phusion PCR Master Mix in a total volume of $25 \mu \mathrm{l}$. The incubation conditions were: 10 cycles of $\left(94^{\circ} \mathrm{C}, 35 \mathrm{~s} ; 65^{\circ} \mathrm{C}\right.$, $30 \mathrm{~s} ; 72^{\circ} \mathrm{C}, 60 \mathrm{~s}$ ). The annealed DNAs were then amplified using the hBak forward and reverse primers. Reaction mixtures contained $3 \mu \mathrm{l}$ annealed DNA, $0.5 \mu \mathrm{M}$ of each primer and $1 \times$ Phusion PCR Master Mix in a total volume of $50 \mu \mathrm{l}$. The cycling conditions were: $94^{\circ} \mathrm{C}, 2 \mathrm{~min} ; 25$ cycles of $\left(94^{\circ} \mathrm{C}, 35 \mathrm{~s} ; 58^{\circ} \mathrm{C}, 30 \mathrm{~s} ; 72^{\circ} \mathrm{C}\right.$, $60 \mathrm{~s}) ; 72^{\circ} \mathrm{C}, 5 \mathrm{~min}$. 
a

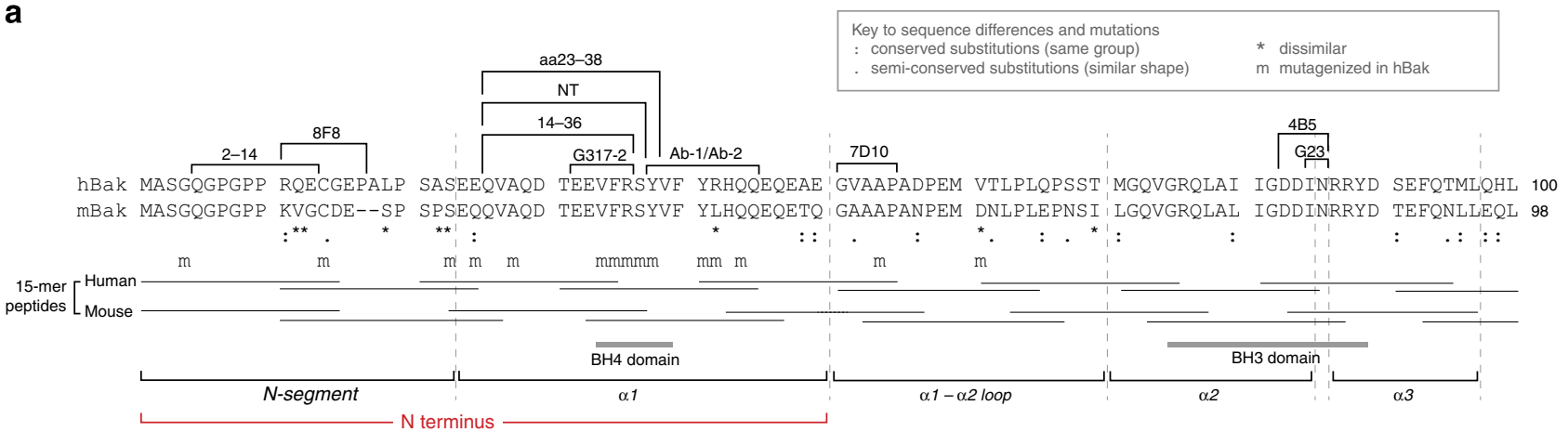

b
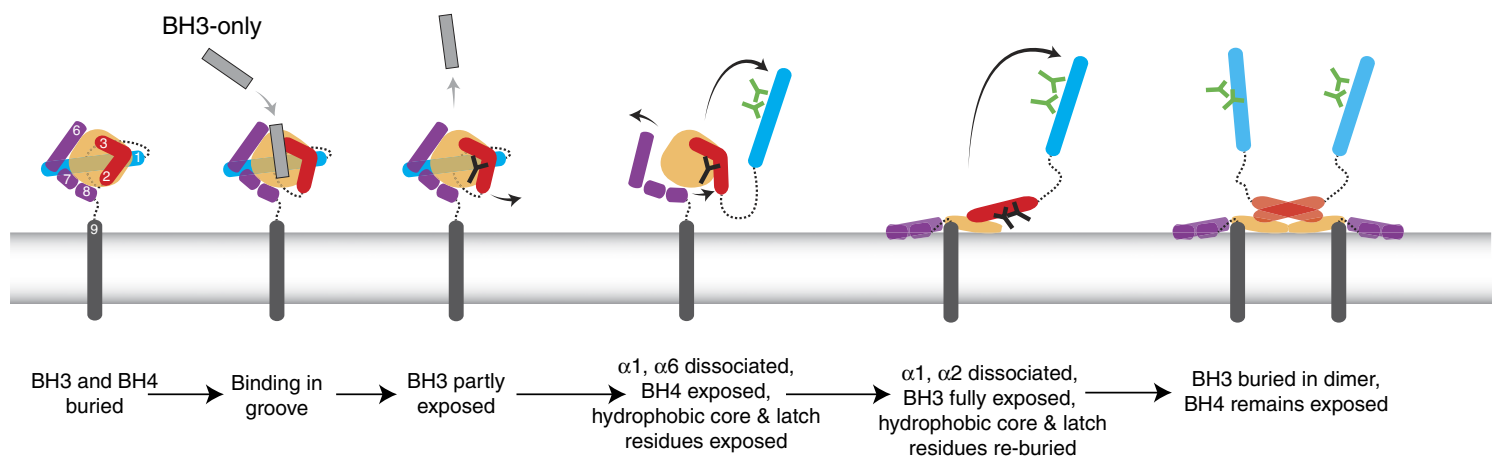

Figure 9 | Summary of epitope mapping data and sequence of conformation changes during Bak activation. (a) Diagram showing the position of Bak antibody epitopes, 15-mer peptides (mouse and human), amino acid differences, mutagenized residues and structural and functional domains in the first 100 residues of Bak. (b) Diagram showing the sequence of conformation changes that Bak undergoes during activation. The $\alpha 1$ (blue), core (orange/red), latch domain (purple) and transmembrane domain (dark grey) are illustrated. $\mathbf{Y}$ indicates an epitope is partially accessible to antibody, that is, 4B5 in black, or G317-2 or Ab-1 in green. YY indicates an epitope is fully accessible to antibody. Bak activation is initiated by transient binding of $\mathrm{BH} 3$-only domains to the hydrophobic groove, provoking movement of $\alpha 2$ and BH3 exposure. Movement of $\alpha 2$ also breaks $\alpha 1$ contact with the $\alpha 6-\alpha 8$ latch, leading to exposure of the $\mathrm{BH} 4$ domain and hydrophobic and aromatic residues in the core and latch domains. Dissociation of $\alpha 1$ from the latch allows $\alpha 2$ to reach a position where the $\mathrm{BH} 3$ domain is fully exposed. Consequently, exposed hydrophobic and aromatic residues in the core and latch become buried in the membrane, or in interactions with other helices to promote formation of symmetric dimers.

Gel-purified reaction products and the PMX-IG vector were digested overnight at $37^{\circ} \mathrm{C}$ with the EcoRI and XhoI restriction enzymes (Roche, \#10703737001 and \#10899194001) in SuRE/Cut Buffer H (Roche, \#11417991001). Gel-purified digested mutant Bak and vector DNAs (1:3 vector:insert ratio) were incubated overnight at room temperature in $1 \times$ ligase buffer containing $2 \mathrm{U}$ T4 DNA ligase (Promega, \#M1801). Ligation products were used to transform electro-competent JM109 cells and colonies grown overnight on LB agar plates supplemented with $100 \mathrm{\mu g} \mathrm{ml}^{-1}$ ampicillin. Subclones were picked, grown overnight and their DNA purified using a QIAprep Spin Mini Kit (QIAGEN, \#27106) and stored at $-20^{\circ} \mathrm{C}$. Positive clones were identified by restriction digestion, confirmed by sequencing and used for retroviral transfection.

Retroviral transfection and transduction. Bak variants were stably expressed in DKO MEF by retroviral infection. pMX-IG constructs were transfected into Phoenix cells using FuGENE6 (Promega, \#E2691) or X-tremeGENE 9 (Roche, \#06365787001) according to the manufacturers' instructions. DKO MEF at $70-80 \%$ confluency in six-well tissue culture plates were transduced with filtered $(0.45 \mu \mathrm{M})$ viral supernatants $(\sim 4 \mathrm{ml} \mathrm{each})$ mixed with $4 \mu \mathrm{g} \mathrm{ml}^{-1}$ polybrene (Sigma, \#107689-10G). After centrifugation $\left(1,083 \mathrm{~g}, 45 \mathrm{~min}, 32^{\circ} \mathrm{C}\right)$, cells were incubated for $24 \mathrm{~h}$, the viral supernatants replaced with fresh media and cells passaged. After a further $24-48 \mathrm{~h}$ at $37^{\circ} \mathrm{C}$, cells were resuspended in buffer (10\% FCS: $90 \%(150 \mathrm{mM}$ $\mathrm{NaCl}, 3.7 \mathrm{mM} \mathrm{KCl}, 2.5 \mathrm{mM} \mathrm{CaCl}_{2}, 1.2 \mathrm{mM} \mathrm{MgSO}{ }_{4}, 14.8 \mathrm{mM}$ HEPES)), filtered and polyclonal GFP-positive populations isolated by FACS using a MoFlo Legacy machine (Beckman Coulter). To prevent infection, sorted cells were cultured in media containing ciprofloxacin (Bayer) for the first 2 weeks.

Peptide scanning arrays. Three sets of N-terminally biotinylated Bak peptides (with SGSG linker sequence) were synthesized by Mimotopes: (i) 21 15-mer, with a five-residue overlap, spanning all residues of mouse Bak; (ii) 21 15-mer, with a fiveresidue overlap, spanning all residues of human Bak; (iii) 39 8-mer, with a oneresidue offset, spanning residues 20-65 of human Bak. Each lyophilized peptide (1-3 mg each) was resuspended in $400 \mu \mathrm{l} 80 \%$ dimethylformamide (DMF) and stored at $-80^{\circ} \mathrm{C}$. 96-well plates (Nunc Immuno Maxisorp, \#442404 or Corning Costar non-treated PVC, \#2797) were coated with $5 \mu \mathrm{g} \mathrm{ml}^{-1}$ streptavidin (Sigma
S-4762) by incubation for $16-24 \mathrm{~h}$ at $37^{\circ} \mathrm{C}$. Plates were washed four times with PBS-T (0.1\% Tween 20 in PBS), blocked by incubating for $1 \mathrm{~h}$ at $\sim 21^{\circ} \mathrm{C}$ in PBS-T containing $0.5 \%(\mathrm{w} / \mathrm{v}) \mathrm{BSA}$ (Sigma) and again washed four times in PBS-T. Peptides (in DMF) were diluted 1:1,000 with $\mathrm{H}_{2} \mathrm{O}$ and $100 \mu \mathrm{l}$ each added to wells of prepared plates. After $1 \mathrm{~h}$ incubation, with shaking, unbound peptides were removed by washing four times with PBS-T. Primary antibodies were diluted (see Table 1) in PBS-T and $100 \mu \mathrm{l}$ incubated with each peptide for at $1 \mathrm{~h}$ at room temperature on a rocking platform. Plates were washed four times with PBS-T and $100 \mu \mathrm{l}$ HRP-conjugated secondary antibody, diluted with PBS-T, was added and plates incubated at room temperature for $1 \mathrm{~h}$ on a rocking platform. Dilutions of secondary antibodies were as for western blots, except for peptide set (iii) where the anti-rabbit and anti-rat antibodies were diluted 1:10,000. Plates were washed four times with PBS-T and bound secondary antibody was detected by incubating with $100 \mu \mathrm{l}$ ABTS buffer (1 mM ABTS (2,2'-Azino-bis(3-ethylbenzthiazoline-6-sulfonic acid)) diammonium salt, Sigma, \#A1888), $100 \mathrm{mM}$ citric acid, $0.03 \% \mathrm{H}_{2} \mathrm{O}_{2}$ ) for 10-45 min and measuring absorbance at $405 \mathrm{nM}$ using a Hidex Chameleon V Multitechnology Platereader.

Bak tethering and activation. MEF were harvested with trypsin, washed with PBS and the outer cell membrane permeabilized by resuspending cells at $1 \times 10^{7} \mathrm{ml}^{-1}$ in ice-cold MELB buffer (20 mM HEPES/NaOH pH 7.5, $100 \mathrm{mM}$ sucrose, $2.5 \mathrm{mM}$ $\mathrm{MgCl}_{2}, 100 \mathrm{mM} \mathrm{KCl}, 1 \times$ complete protease inhibitor (Roche), $4 \mu \mathrm{g} \mathrm{ml}^{-1}$ pepstatin A) containing $0.025 \%$ digitonin. After $5 \mathrm{~min}$ incubation on ice, cell permeabilization was verified by uptake of trypan blue. For cytochrome $c$ release assays and IPs, permeabilized cells were centrifuged $\left(16,200 \mathrm{~g}, 5 \mathrm{~min}, 4^{\circ} \mathrm{C}\right)$ and membrane fractions resuspended in MELB buffer and kept on ice. For enterokinase cleavage assays of Bak, membrane fractions were permeabilized and subsequently resuspended in MELB buffer lacking $1 \times$ complete protease inhibitor.

Intramolecular tethers were induced by disulfide bonding of cysteines using CuPhe diluted 500-fold into the sample. Permeabilized MEF or membrane fractions were incubated with $\mathrm{CuPhe}$ at least $5 \mathrm{~min}$ on ice. The efficiency of tethering was assessed by western blotting after mixing untreated or CuPhe-treated samples with an equal volume of sample buffer $(0.15 \mathrm{M}$ Tris $\mathrm{pH} 6.8,30 \%$ glycerol, $1.2 \% \mathrm{SDS}, 0.018 \mathrm{mg} \mathrm{ml}^{-1}$ bromophenol blue) containing $25 \mathrm{mM}$ EDTA to chelate the copper. 
To induce Bak activation, untreated or CuPhe-treated samples were incubated with $100 \mathrm{nM}$ caspase- 8 cleaved human Bid or $10 \mu \mathrm{M}$ Bim BH3 peptide for $30 \mathrm{~min}$ at $30^{\circ} \mathrm{C}$, or for $30 \mathrm{~min}$ at $44^{\circ} \mathrm{C}$. Activation reactions were stopped by placing samples on ice for at least $5 \mathrm{~min}$.

Cytochrome $\mathrm{c}$ release. Following activation of Bak in membrane fractions, the samples were centrifuged at $16,200 \mathrm{~g}, 5 \mathrm{~min}, 4^{\circ} \mathrm{C}$. The resulting supernatant and pellet fractions were each mixed with sample buffer containing $5 \%(\mathrm{v} / \mathrm{v})$ 2-mercaptoethanol (2Me) and western blotting performed for cytochrome $c$.

Immunoprecipitation of activated Bak. Following activation of Bak in membrane fractions, samples were solubilized by incubation with $1 \%$ digitonin on ice for at least $30 \mathrm{~min}$. Samples were centrifuged $\left(16,200 \mathrm{~g}, 5 \mathrm{~min}, 4^{\circ} \mathrm{C}\right)$ and supernatants precleared by $30 \mathrm{~min}$ incubation at $4^{\circ} \mathrm{C}$ with Protein G Sepharose beads 4 Fast Flow (GE Healthcare Life Sciences), pre-washed with Onyx buffer $(20 \mathrm{mM} \mathrm{TrisCl} \mathrm{pH}$ 7.4, $135 \mathrm{mM} \mathrm{NaCl}, 1.5 \mathrm{mM} \mathrm{MgCl}_{2}, 1 \mathrm{mM}$ EGTA, $10 \%$ glycerol, $1 \times$ complete protease inhibitor, $4 \mu \mathrm{g} \mathrm{ml}^{-1}$ pepstatin A). After removing beads by repeated washes in Onyx buffer, lysates were incubated with constant agitation for $2 \mathrm{~h}$ at $4^{\circ} \mathrm{C}$ with $4 \mu \mathrm{g} \mathrm{ml}^{-1}$ anti-Bak antibody, followed by incubation for $1 \mathrm{~h}$ at $4{ }^{\circ} \mathrm{C}$ with additional pre-washed Sepharose $\mathrm{G}$ beads. Immune complexes were isolated by centrifugation $\left(16,200 \mathrm{~g}, 2 \mathrm{~min}, 4^{\circ} \mathrm{C}\right)$, washed four times with Onyx buffer, resuspended in sample buffer containing $5 \%(\mathrm{v} / \mathrm{v}) 2 \mathrm{Me}$ and analysed by western blotting.

FACS analysis. Following activation of Bak in permeabilized cells, the samples were centrifuged $\left(900 \mathrm{~g}, 3 \mathrm{~min}, 4^{\circ} \mathrm{C}\right)$, cells were washed with FACS buffer $(10 \%$ FBS: $90 \%\left(1.2 \mathrm{mM} \mathrm{MgSO}_{4}, 7.4 \mathrm{mM}\right.$ HEPES-NaOH, $0.8 \mathrm{mM} \mathrm{K}_{2} \mathrm{HPO}_{4}, 140 \mathrm{mM}$ $\mathrm{NaCl})$ ) and incubated $40-70$ min on ice with anti-Bak antibodies (Ab-1, G317-2 or 4B5) diluted 1:100 in FACS buffer. To assess BH3 domain exposure during Bak activation, $10 \mu \mathrm{g} \mathrm{ml}^{-1}$ anti-Bak (4B5) antibody was added to untreated and CuPhe-treated samples immediately before the Bak activation step. After incubation with primary antibody, cells were washed with FACS buffer and incubated 40-70 min on ice with RPE-labelled secondary antibody diluted 1:200 in FACS buffer. The cells were washed with FACS buffer and data collected immediately using an LSRII flow cytometer (BD) fitted with FACSDiVa software (BD) were subsequently analysed using WEASEL (v2.7).

Doublets and debris were routinely excluded by gating using forward scatter cytometry and side scatter cytometry. To quantify the proportions of cells with exposed $\mathrm{BH} 4$ or $\mathrm{BH} 3$ domains, a marker was positioned to distinguish negatively and positively staining populations, with untreated cells (grey histograms) used as guides for negative staining. The MFI of cells in positive-staining populations was also compared in cases where the proportions of cells appeared similar but the amount of staining (that is, profile position on $x$ axis) differed in tethered versus untethered cells.

Bak cleavage by EK. Following tethering and/or activation of Bak in membrane fractions, samples were incubated with or without recombinant EK ( 3 or 4.4 units per $50 \mu \mathrm{l}$ sample; Merck \#69066-3) for $2 \mathrm{~h}$ at room temperature. EK was inactivated by the addition of PMSF $(4 \mathrm{mM})$ and cleavage assessed by western blotting after mixing samples with an equal volume of sample buffer containing 5\% (v/v) $2 \mathrm{Me}$.

Blue Native-PAGE. Blue Native-PAGE was performed after Bak activation in permeabilized MEF (as described above) using the Invitrogen NativePAGE Novex Bis-Tris Gel System. All samples were incubated $30 \mathrm{~min}$ on ice, centrifuged $\left(16,200 \mathrm{~g}, 5 \mathrm{~min}, 4^{\circ} \mathrm{C}\right)$ and the pellets resuspended in Solubilization buffer $(20 \mathrm{mM}$ Bis-Tris pH 7.4, $50 \mathrm{mM} \mathrm{NaCl}, 10 \%$ glycerol, $1 \%$ digitonin). After at least $30 \mathrm{~min}$ on ice, the samples were centrifuged $\left(16,200 \mathrm{~g}, 5 \mathrm{~min}, 4^{\circ} \mathrm{C}\right)$, the supernatants mixed with NativePAGE sample buffer and NativePAGE 5\% G-250 sample additive and proteins separated on $4-16 \%$ NativePAGE Novex Bis-Tris gels in chambers containing $1 \times$ NativePAGE anode buffer and $1 \times$ NativePAGE cathode buffer containing $1 \times$ NativePAGE cathode additive. After running at $150 \mathrm{~V}$ for 30 $45 \mathrm{~min}$, the blue cathode buffer was replaced with $1 \times$ NativePAGE cathode buffer without any cathode additive and gels run for a further $80-95$ min. Proteins were transferred to polyvinylidene difluoride membranes at $40 \mathrm{~V}$ for $150 \mathrm{~min}$ in buffer containing $25 \mathrm{mM}$ Tris, $192 \mathrm{mM}$ glycine, $20 \%$ methanol and $0.037 \%$ SDS. Membranes were de-stained by $\sim 40 \mathrm{~min}$ incubation in fixative [10\% acetic acid, $45 \%$ methanol]. After washing 3 times for $10 \mathrm{~min}$ in water, membranes were incubated in blocking solution and proteins detected by Western blotting. To compare sample loading, excess sample was mixed 1:1 with sample buffer containing $5 \%(\mathrm{v} / \mathrm{v}) 2 \mathrm{Me}$ and analysed by western blotting.

Western blotting. Samples were heated $5 \mathrm{~min}$ at $>95^{\circ} \mathrm{C}$, spun briefly and proteins separated by SDS-PAGE using pre-cast $12 \%$ TGX gels (Bio-Rad) and transferred to nitrocellulose membranes at $40 \mathrm{~V}$ for $150 \mathrm{~min}$ in buffer containing $25 \mathrm{mM}$ Tris, $192 \mathrm{mM}$ glycine and $20 \%$ methanol. Note that transfer of proteins to polyvinylidene difluoride rather than nitrocellulose membranes resulted in inferior signals for most Bak antibodies (data not shown). Nonspecific binding of antibodies was blocked by incubation for $30-45$ min with $5 \%$ nonfat milk powder in
TBS (20 mM TrisHCl pH7.6, $137 \mathrm{mM} \mathrm{NaCl}$ ) with $0.1 \%$ Tween 20. Membranes were rinsed with TBS/0.1\% Tween 20 and incubated with primary antibodies at room temperature for $1-5 \mathrm{~h}$ or at $4{ }^{\circ} \mathrm{C}$ overnight. The $\mathrm{Ab}-1, \mathrm{Ab}-2$ and $4 \mathrm{~B} 5$ anti-Bak antibodies and anti-cytochrome $c$ antibody were diluted in TBS/ $0.05 \%$ Tween 20 , anti- $\beta$-actin antibody was diluted in TBS $/ 5 \%$ BSA. All other primary antibodies were diluted in blocking solution. Membranes were washed three times (5 min each) in TBS $/ 0.1 \%$ Tween 20 and incubated $1-2 \mathrm{~h}$ at room temperature with secondary antibody diluted in blocking solution, except for cytochrome $c$ blots where the anti-mouse secondary was also diluted in TBS/0.05\% Tween 20 . Membranes were washed three times ( $5 \mathrm{~min}$ each) in TBS/0.1\% Tween 20 , developed with Luminata Forte HRP substrate (Millipore) and bioluminescent signals detected using a ChemiDoc XRS + System fitted with ImageLab software (Bio-Rad). Uncropped images from Figs 1-4, 7 and 8 are shown in Supplementary Figs 8-12.

Structural modelling. The inactive Bak structure $2 \mathrm{IMS}^{3}$ was downloaded from PDB and manipulated using PyMOL (DeLano Scientific LLC). The images were saved as PNG files.

\section{References}

1. Czabotar, P. E., Lessene, G., Strasser, A. \& Adams, J. M. Control of apoptosis by the BCL-2 protein family: implications for physiology and therapy. Nat. Rev. Mol. Cell. Biol. 15, 49-63 (2014).

2. Westphal, D., Kluck, R. M. \& Dewson, G. Building blocks of the apoptotic pore: how Bax and Bak are activated and oligomerize during apoptosis. Cell Death Differ. 21, 196-205 (2014).

3. Moldoveanu, T. et al. The X-ray structure of a BAK homodimer reveals an inhibitory zinc binding site. Mol. Cell 24, 677-688 (2006).

4. Suzuki, M., Youle, R. J. \& Tjandra, N. Structure of Bax: coregulation of dimer formation and intracellular localization. Cell 103, 645-654 (2000).

5. Ferrer, P. E., Frederick, P., Gulbis, J. M., Dewson, G. \& Kluck, R. M. Translocation of a Bak C-terminus mutant from cytosol to mitochondria to mediate cytochrome $\mathrm{C}$ release: implications for Bak and Bax apoptotic function. PLoS ONE 7, e31510 (2012).

6. Setoguchi, K., Otera, H. \& Mihara, K. Cytosolic factor- and TOM-independen import of C-tail-anchored mitochondrial outer membrane proteins. EMBO J. 25, 5635-5647 (2006).

7. Nechushtan, A., Smith, C. L., Hsu, Y. T. \& Youle, R. J. Conformation of the Bax C-terminus regulates subcellular location and cell death. EMBO J. 18, 2330-2341 (1999).

8. Lovell, J. F. et al. Membrane binding by tBid initiates an ordered series of events culminating in membrane permeabilization by Bax. Cell 135, 1074-1084 (2008).

9. Dewson, G. et al. To trigger apoptosis Bak exposes its $\mathrm{BH} 3$ domain and homo-dimerizes via BH3:grooove interactions. Mol. Cell 30, 369-380 (2008).

10. Griffiths, G. J. et al. Cell damage-induced conformational changes of the pro-apoptotic protein Bak in vivo precede the onset of apoptosis. J. Cell Biol. 144, 903-914 (1999)

11. Wei, M. C. et al. tBID, a membrane-targeted death ligand, oligomerizes BAK to release cytochrome c. Genes Dev. 14, 2060-2071 (2000).

12. Moldoveanu, T. et al. BID-induced structural changes in BAK promote apoptosis. Nat. Struct. Mol. Biol. 20, 589-597 (2013).

13. Cuconati, A., Mukherjee, C., Perez, D. \& White, E. DNA damage response and MCL-1 destruction initiate apoptosis in adenovirus-infected cells. Genes Dev. 17, 2922-2932 (2003).

14. Dewson, G. et al. Bak activation for apoptosis involves oligomerization of dimers via their alpha6 helices. Mol. Cell 36, 696-703 (2009).

15. Kvansakul, M. et al. Vaccinia virus anti-apoptotic F1L is a novel Bcl-2-like domain-swapped dimer that binds a highly selective subset of $\mathrm{BH}$-containing death ligands. Cell Death Differ. 15, 1564-1571 (2008).

16. Dewson, G. et al. Bax dimerizes via a symmetric BH3:groove interface during apoptosis. Cell Death Differ. 19, 661-670 (2012).

17. Hsu, Y. T. \& Youle, R. J. Nonionic detergents induce dimerization among members of the Bcl-2 family. J. Biol. Chem. 272, 13829-13834 (1997).

18. Westphal, D., Dewson, G., Czabotar, P. E. \& Kluck, R. M. Molecular biology of Bax and Bak activation and action. Biochim. Biophys. Acta 1813, 521-531 (2011).

19. Czabotar, P. E. et al. Bax crystal structures reveal how $\mathrm{BH} 3$ domains activate bax and nucleate its oligomerization to induce apoptosis. Cell 152, 519-531 (2013).

20. Brouwer, J. M. et al. Bak core and latch domains separate during activation, and freed core domains form symmetric homodimers. Mol. Cell 55, 938-946 (2014).

21. Bleicken, S. et al. Structural model of active bax at the membrane. Mol. Cell 56, 496-505 (2014).

22. Oh, K. J. et al. Conformational changes in BAK, a pore-forming proapoptotic $\mathrm{Bcl}-2$ family member, upon membrane insertion and direct evidence for the 
existence of $\mathrm{BH} 3-\mathrm{BH} 3$ contact interface in BAK homo-oligomers. J. Biol. Chem. 285, 28924-28937 (2010).

23. Cheng, E. H., Sheiko, T. V., Fisher, J. K., Craigen, W. J. \& Korsmeyer, S. J. VDAC2 inhibits BAK activation and mitochondrial apoptosis. Science 301, 513-517 (2003).

24. Geysen, H. M., Rodda, S. J., Mason, T. J., Tribbick, G. \& Schoofs, P. G. Strategies for epitope analysis using peptide synthesis. J. Immunol. Methods 102, 259-274 (1987).

25. Weber, K., Harper, N., Schwabe, J. \& Cohen, G. M. BIM-mediated membrane insertion of the BAK pore domain is an essential requirement for apoptosis. Cell Rep. 5, 409-420 (2013).

26. Aluvila, S. et al. Organization of the mitochondrial apoptotic BAK pore: oligomerization of the BAK homodimers. J. Biol. Chem. 289, 2537-2551 (2014).

27. Llambi, F. et al. A unified model of mammalian BCL-2 protein family interactions at the mitochondria. Mol. Cell 44, 517-531 (2011).

28. Ruffolo, S. C. \& Shore, G. C. BCL-2 selectively interacts with the BID-induced open conformer of BAK, inhibiting BAK auto-oligomerization. J. Biol. Chem. 278, 25039-25045 (2003).

29. Lazarou, M. et al. Inhibition of Bak activation by VDAC2 is dependent on the Bak transmembrane anchor. J. Biol. Chem. 285, 36876-36883 (2010).

30. George, N. M., Evans, J. J. \& Luo, X. A three-helix homo-oligomerization domain containing $\mathrm{BH} 3$ and $\mathrm{BH} 1$ is responsible for the apoptotic activity of Bax. Genes Dev. 21, 1937-1948 (2007)

31. Fletcher, J. I. et al. Apoptosis is triggered when prosurvival Bcl-2 proteins cannot restrain Bax. Proc. Natl Acad. Sci. USA 105, 18081-18087 (2008).

32. Ding, J. et al. After embedding in membranes antiapoptotic Bcl-XL protein binds both $\mathrm{Bcl}-2$ homology region 3 and helix 1 of proapoptotic Bax protein to inhibit apoptotic mitochondrial permeabilization. J. Biol. Chem. 289, 11873-11896 (2014)

33. Tompa, P. et al. On the sequential determinants of calpain cleavage. J. Biol. Chem. 279, 20775-20785 (2004)

34. Peyerl, F. W. et al. Elucidation of some Bax conformational changes through crystallization of an antibody-peptide complex. Cell Death Differ. 14, 447-452 (2007).

35. Hsu, Y.-T. \& Youle, R. J. Bax in murine thymus is a soluble monomeric protein that displays differential detergent-induced conformations. J. Biol. Chem. 273, 10777-10783 (1998)

36. Desagher, S. et al. Bid-induced conformational change of Bax is responsible for mitochondrial cytochrome $\mathrm{c}$ release during apoptosis. J. Cell Biol. 144, 891-901 (1999).

37. Gavathiotis, E., Reyna, D. E., Davis, M. L., Bird, G. H. \& Walensky, L. D. $\mathrm{BH} 3$-triggered structural reorganization drives the activation of proapoptotic BAX. Mol. Cell 40, 481-492 (2010).
38. Chen, L. et al. Differential targeting of prosurvival Bcl-2 proteins by their BH3-only ligands allows complementary apoptotic function. Mol. Cell 17, 393-403 (2005).

39. Shoemaker, R. H. The NCI60 human tumour cell line anticancer drug screen. Nat. Rev. Cancer 6, 813-823 (2006).

40. Tran, V. H. et al. Bak apoptotic function is not directly regulated by phosphorylation. Cell Death Dis. 4, e452 (2013).

41. Xiao, Y., Zhong, Y., Greene, W., Dong, F. \& Zhong, G. Chlamydia trachomatis infection inhibits both Bax and Bak activation induced by staurosporine. Infect. Immun. 72, 5470-5474 (2004).

42. Krajewski, S., Krajewska, M. \& Reed, J. C. Immunohistochemical analysis of in vivo patterns of Bak expression, a proapoptotic member of the Bcl-2 protein family. Cancer Res. 56, 2849-2855 (1996).

\section{Acknowledgements}

We thank P. Colman for discussion and critical comments on the manuscript. Our work is supported by the Australian Research Council (FT100100754 and FT100100791), the National Health and Medical Research Council of Australia (NHMRC: 1008434), the Victorian State Government Operational Infrastructure Support grant and the Australian Government NHMRC Independent Research Institutes Infrastructure Support Scheme.

\section{Author contributions}

A.E.A. and S.C.F. performed western blotting, peptide array and immunoprecipitation analyses. A.E.A. and I.K.L.T. performed tethering. A.E.A. performed FACS analyses and Blue-Native-PAGE. R.C.B. and G.D. generated Bak mutants and associated cell lines. A.E.A. and R.M.K. wrote the manuscript.

\section{Additional information}

Supplementary Information accompanies this paper at http://www.nature.com/ naturecommunications

Competing financial interests: The authors declare no competing financial interests

Reprints and permission information is available online at http://npg.nature.com/ reprintsandpermissions/

How to cite this article: Alsop, A. E. et al. Dissociation of Bak $\alpha 1$ helix from the core and latch domains is required for apoptosis. Nat. Commun. 6:6841 doi: 10.1038/ncomms7841 (2015) 